\title{
Molecular Structure, Vibrational Spectra and Docking Studies of Abacavir by Density Functional Theory
}

\author{
R. Solaichamy, J. Karpagam \\ Department of physics (Engg), Annamalai University, Annamalainagar-608 002, Tamil Nadu, India. \\ * jkarpagamphd@gmail.com, Mobile: +91 9944721275.
}

Keywords: Abacavir, FT-IR, FT-Raman, NBO, Molecular Docking, DFT.

\begin{abstract}
In this study, optimized geometry, spectroscopic (FT-IR, FT-Raman, UV) analysis, and electronic structure analysis of Abacavir were investigated by utilizing DFT/B3LYP with $6-31 G(d, p)$ as a basis set. Complete vibrational assignments and correlation of the fundamental modes for the title compound were carried out. The calculated molecular geometry has been compared with available X-ray data of Abacavir. The calculated HOMO and LUMO energies show that charge transfer occurs within the molecule. The molecular stability and bond strength have been investigated by applying the Natural Bond Orbital (NBO) analysis. The computational molecular docking studies of title compound have been performed.
\end{abstract}

\section{Introduction}

The managing of human immunodeficiency virus (HIV) includes the use of multiple antiretroviral, since single drug therapy becomes ineffective due to development of HIV resistant strains. According to treatment guidelines antiretroviral regimen should contain at least two nucleoside analogue reverse transcriptase inhibitors (NRTIs) and one non-nucleoside reverse transcriptase inhibitor (NNRTI) in a fixed dose combination. This is very efficient in the treatment of HIV. Abacavir (ABC) is a nucleoside reverse transcriptase inhibitor (NRTI) with activity against Human Immunodeficiency Virus Type 1 (HIV-1). ABC is phosphorylated to active metabolites that compete for incorporation into viral DNA. Intracellularly, ABC is converted by cellular enzymes to the active metabolite carbovir triphosphate, an analogue of deoxyguanosine-50-triphosphate (dGTP). Chemically ABC is [(1S,4R)-4-[2-amino-6-(cyclopropylamino)-9H-purin-9-yl] cyclopent2-en-1-yl] methanol [1,2]. The introduction of highly active antiretroviral therapy (HAART) has profoundly altered both the course and prognosis of HIV infection. After 1996, the availability of protease inhibitors (PI) transformed HIV-infection from a progressive and almost uniformly fatal condition to a treatable chronic infection. Choosing an initial antiretroviral regimen is one of the most important decisions faced by clinicians managing HIV disease. Several antiretroviral combinations have proven sufficiently potent to achieve viral suppression in most treated patients. However, maintaining efficacy depends on other factors, such as the durability of antiviral suppression, tolerability, risk of long-term toxicity, and patient convenience [3].

The present work mainly deals with detailed structural conformation, experimental FT-IR and FT-Raman spectra, vibrational assignments using total energy distribution (TED) and Molecular docking activity as well as DFT/B3LYP calculations for ABC. Vibrational spectra of Abacavir have been analyzed on the basis of calculated total energy distribution (TED). Theoretically computed vibrational wavenumbers were compared with experimental values. The natural bond orbital (NBO) analysis can be employed to identify and substantiate the possible intra and intermolecular interactions between the units that would form the H-bonded network. The UVVis spectroscopic studies along with HOMO-LUMO analysis have been used to explain the charge transfer within the molecule.

\section{Experimental details}

The compound Abacavir was purchased from Aldrich chemicals, USA and used as such to record the FT-IR and FT-Raman, UV spectra. The FT-IR spectrum of Abacavir compound was 
recorded in the range of $4000-400 \mathrm{~cm}^{-1}$ on a BRUKER Optik GmbH FT-IR spectrometer using $\mathrm{KBr}$ pellet technique. The spectrum was recorded in the room temperature, with scanning speed of $10 \mathrm{~cm}^{-1}$, and spectral resolution: $4 \mathrm{~cm}^{-1}$. FT-Raman spectrum of the title compound was recorded using $1064 \mathrm{~nm}$ line of $\mathrm{Nd}$ :YAG laser as excitation wavelength in the region $3500-50 \mathrm{~cm}^{-1}$ on a BRUKER RFS 27: FT-Raman Spectrometer equipped with FT-Raman molecule accessory. The spectral resolution was set to $2 \mathrm{~cm}^{-1}$ in back scattering mode. The laser output was kept at $100 \mathrm{~mW}$ for the solid sample. The ultraviolet absorption spectra of ABC were examined in the range 200$800 \mathrm{~nm}$ using Cary $500 \mathrm{UV}-$ VIS-NIR spectrometer. The UV pattern is taken from a 10 to $5 \mathrm{M}$ solution of $\mathrm{ABC}$, dissolved in ethanol solvent. The theoretically predicted IR and Raman spectra at B3LYP/6-31G(d,p) level calculation along with experimental FT-IR and FT-Raman spectra are shown in Fig. 2 and 3.

\section{Computational details}

The density functional theory DFT/B3LYP with the 6-31G(d,p) as basis set was adopted to calculate the properties of Abacavir in the present work. All the calculations were performed using Gaussian 03W program package [4] with the default convergence criteria without any constraint on the geometry [5]. The assignments of the calculated wavenumbers are aided by the animation option of Gauss View 5.0 graphical interface for Gaussian programs, which gives a visual presentation of the shape of the vibrational modes along with available related molecules [6]. Furthermore, theoretical vibrational spectra of the title compound were interpreted by means of TED using the VEDA 4 program [7]. The optimized structural parameters were used in the vibrational frequency calculations at DFT levels to characterize all stationary points as minima. As the hybrid B3LYP functional tends to overestimate the fundamental normal modes of vibration, the computed frequencies were scaled with appropriate values to bring harmonization between the theoretical and experimental wavenumbers [8]. Vibrational frequencies were computed at DFT level which had reliable one-to-one correspondence with experimental IR and Raman frequencies [9]. The Natural Bond Orbital (NBO) calculations were performed using NBO 3.1 program [10] as implemented in the Gaussian 03W [4] package at the DFT/B3LYP level; in order to understand various second order interactions between filled orbital of one subsystem and vacant orbital of another subsystem which is a measure of the intermolecular delocalization or hyper conjugation.

\subsection{Prediction of Raman intensities}

The Raman activities $\left(\mathrm{S}_{\mathrm{Ra}}\right)$ calculated with Gaussian 03W program [4] converted to relative Raman intensities $\left(\mathrm{I}_{\mathrm{Ra}}\right)$ using the following relationship derived from the intensity theory of Raman scattering [11]

$$
I_{i}=\frac{f\left(v_{o}-v_{i}\right)^{4} S_{i}}{v_{i}\left[1-\exp \left(-h c v_{i} / k t\right)\right]}
$$

where, $v_{0}$ is the laser exciting wavenumber in $\mathrm{cm}^{-1}$ (in this work, we have used the excitation wavenumber $v_{0}=9398.5 \mathrm{~cm}^{-1}$, which corresponds to the wavelength of $1064 \mathrm{~nm}$ of an Nd-YAG laser), $v_{i}$ the vibrational wavenumber of the $i^{\text {th }}$ normal mode $\left(\mathrm{cm}^{-1}\right)$ while $S_{i}$ is the Raman scattering activity of the normal mode $v_{\mathrm{i}}[12]$.

\subsection{Docking Studies}

The molecular structure of protein (PDB ID: 3VRI) was taken from RCSB Protein Data Bank, http://www.rcsb.org/pdb [13]. Initial structures of Abacavir were generated by ChemBioOffice 2008. The geometries of Abacavir legand were subsequently optimized at DFT/B3LYP/ 6-31G (d,p) by Gaussian 03 [4]. The molecular modeling docking calculations of Abacavir legand with 3VRI protein were carried out by means of the Autodock tools (ADT) v1.5.4 [14] and Autodock 4.2.3 program from the Scripps Research Institute. In docking study, the search was extended over the whole receptor Abacavir used as blind docking. The grid maps were generated with $0.375 \AA$ spaces using a grid box of 70-70-70 $\AA$. The search was carried out with the 
Lamarckian Genetic Algorithm because it has been pointed out to be most efficient, reliable and successful methods in Autodock [15]. The docking parameters used were as follows: GA population size $=150$; maximum number of energy evaluation $=25,00,000$ and others used were default parameters. The docking conformation with the lowest binding free energy was used for further analysis by Molegro Molecular Viewer software from http:// www.clcbio.com/products/molegro/ [16].

\section{Results and Discussions}

\subsection{Structural analysis}

The optimized geometric parameters such as bond lengths, bond angles and dihedral angles of the title molecule were given in Table 1 using DFT calculation with 6-31G(d,p) as a basis set. The atom numbering scheme of the title compound adopted in this study is given in Fig. 1. To the best of our knowledge, experimental data on the geometric structure of the title molecule are not available till date in the literature. Our molecule Abacavir is compared with XRD data of closely related molecules Abacavir methanol 2.5-solvate [17]. The $\mathrm{C}-\mathrm{C}$ bond length of the purine ring found at $\mathrm{C} 11-\mathrm{C} 16=1.396 \AA$ and $\mathrm{C} 15-\mathrm{C} 16=1.414 \AA$ calculated from DFT method which is agree well with XRD value at $1.383 \AA$ and $1.412 \AA$ respectively. The $\mathrm{C}-\mathrm{N}$ bond length of the purine ring varies from $1.338 \AA$ to $1.335 \AA$ by DFT method $1.334 \AA$ to $1.370 \AA$ by XRD respectively. Similarly, in the cyclopentane ring C-C bond length varies in the range $1.334 \AA$ to $1.556 \AA$ by DFT and $1.329 \AA$ to $1.550 \AA$ by XRD respectively also $\mathrm{C}-\mathrm{H}$ bond length of this ring found from $1.086 \AA$ to $1.100 \AA$ by DFT and $0.990 \AA$ to $1.000 \AA$ by XRD respectively this is correlate well with calculated values as well as literature data [18]. Methanol group C-H bond lengths are C1$\mathrm{H} 22=1.102 \AA$ (DFT), $0.990 \AA$ (XRD) and $\mathrm{C} 1-\mathrm{H} 23=1.095 \AA$ (DFT), 0.990 (XRD). The cyclopropylamine group bond length has been found at $\mathrm{C} 18-\mathrm{C} 19=1.507 \AA$ (DFT), $1.503 \AA$ (XRD) and $\mathrm{C} 18-\mathrm{C} 20=1.506 \AA$ (DFT), $1.493 \AA$ (XRD). The N-H bond length of the amino and ammine group is $\mathrm{N} 21-\mathrm{H} 38=1.008 \AA$ and $\mathrm{N} 21-\mathrm{H} 39=1.008 \AA$ by DFT method and observed by XRD and $\mathrm{N} 17-\mathrm{H} 32=1.011 \AA$ (DFT), $0.880 \AA$ (XRD) respectively. The $\mathrm{O} 2-\mathrm{H} 24$ bond length found at $0.965 \AA$ by DFT method and $0.839 \AA$ by XRD.

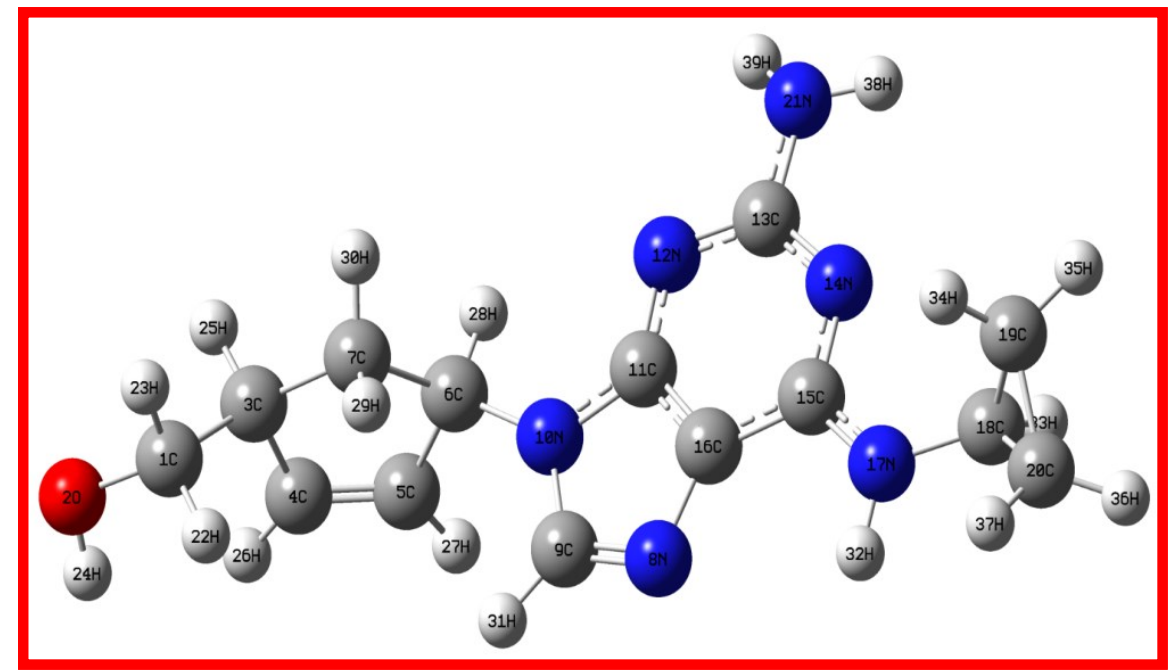

Figure 1. Optimized Molecular structure and atomic numbering of Abacavir. 
Table 1. Comparison of experimental and theoretical optimized parameter values of the Abacavir [bond length in $\left(\mathrm{A}^{\circ}\right)$, angles in $\left(^{\circ}\right)$ ].

\begin{tabular}{|c|c|c|c|c|c|c|c|c|}
\hline Bond length & B3LYP & $\operatorname{Exp}^{a}$ & Bond angle & B3LYP & $\operatorname{Exp}^{a}$ & Dihedral angle & B3LYP & $\operatorname{Exp}^{\mathrm{a}}$ \\
\hline $\mathrm{C} 1-\mathrm{O} 2$ & 1.421 & 1.419 & $\mathrm{O} 2-\mathrm{C} 1-\mathrm{C} 3$ & 113.2 & 111.1 & $\mathrm{C} 3-\mathrm{C} 1-\mathrm{O} 2-\mathrm{H} 24$ & 74.28 & \\
\hline C1-C3 & 1.537 & 1.526 & $\mathrm{O} 2-\mathrm{C} 1-\mathrm{H} 22$ & 111.5 & 109.4 & $\mathrm{H} 22-\mathrm{C} 1-\mathrm{O} 2-\mathrm{H} 24$ & -49.62 & \\
\hline C1-H22 & 1.102 & 0.990 & $\mathrm{O} 2-\mathrm{C} 1-\mathrm{H} 23$ & 105.9 & 109.4 & $\mathrm{H} 23-\mathrm{C} 1-\mathrm{O} 2-\mathrm{H} 24$ & -165.96 & \\
\hline $\mathrm{C} 1-\mathrm{H} 23$ & 1.095 & 0.990 & $\mathrm{C} 3-\mathrm{C} 1-\mathrm{H} 22$ & 109.4 & 109.4 & $\mathrm{O} 2-\mathrm{C} 1-\mathrm{C} 3-\mathrm{C} 4$ & -65.38 & 64.7 \\
\hline $\mathrm{O} 2-\mathrm{H} 24$ & 0.965 & 0.839 & $\mathrm{C} 3-\mathrm{C} 1-\mathrm{H} 23$ & 109.3 & 109.4 & $\mathrm{O} 2-\mathrm{C} 1-\mathrm{C} 3-\mathrm{C} 7$ & 177.31 & 179.07 \\
\hline $\mathrm{C} 3-\mathrm{C} 4$ & 1.514 & 1.495 & $\mathrm{H} 22-\mathrm{C} 1-\mathrm{H} 23$ & 107.3 & 108.0 & $\mathrm{O} 2-\mathrm{C} 1-\mathrm{C} 3-\mathrm{H} 25$ & 55.35 & \\
\hline $\mathrm{C} 3-\mathrm{C} 7$ & 1.552 & 1.554 & $\mathrm{C} 1-\mathrm{O} 2-\mathrm{H} 24$ & 107.9 & 109.4 & $\mathrm{H} 22-\mathrm{C} 1-\mathrm{C} 3-\mathrm{C} 4$ & 59.63 & \\
\hline $\mathrm{C} 3-\mathrm{H} 25$ & 1.100 & 1.000 & $\mathrm{C} 1-\mathrm{C} 3-\mathrm{C} 4$ & 114.6 & 109.7 & $\mathrm{H} 22-\mathrm{C} 1-\mathrm{C} 3-\mathrm{C} 7$ & -57.67 & \\
\hline C4-C5 & 1.334 & 1.329 & C1-C3-C7 & 113.0 & 112.7 & H22-C1-C3-H25 & -179.63 & \\
\hline $\mathrm{C} 4-\mathrm{H} 26$ & 1.087 & 0.950 & C1-C3-H25 & 106.7 & 110.3 & H23-C1-C3-C4 & 176.83 & \\
\hline C5-C6 & 1.513 & 1.507 & C4-C3-C7 & 102.8 & 103.2 & $\mathrm{H} 23-\mathrm{C} 1-\mathrm{C} 3-\mathrm{C} 7$ & 59.53 & \\
\hline $\mathrm{C} 5-\mathrm{H} 27$ & 1.086 & 0.950 & $\mathrm{C} 4-\mathrm{C} 3-\mathrm{H} 25$ & 109.0 & 110.3 & $\mathrm{H} 23-\mathrm{C} 1-\mathrm{C} 3-\mathrm{H} 25$ & -62.43 & \\
\hline C6-C7 & 1.556 & 1.550 & C7-C3-H25 & 110.8 & 110.3 & C1-C3-C4-C5 & -133.21 & \\
\hline C6-N10 & 1.462 & 1.480 & C3-C4-C5 & 112.8 & 113.6 & C1-C3-C4-H26 & 50.12 & \\
\hline C6-H28 & 1.096 & 1.000 & C3-C4-H26 & 122.7 & 123.2 & C7-C3-C4-C5 & -10.24 & \\
\hline C7-H29 & 1.096 & 0.990 & $\mathrm{C} 5-\mathrm{C} 4-\mathrm{H} 26$ & 124.3 & 123.2 & C7-C3-C4-H26 & 173.08 & \\
\hline C7-H30 & 1.092 & 0.990 & C4-C5-C6 & 111.8 & 111.3 & H25-C3-C4-C5 & 107.38 & \\
\hline N8-C9 & 1.311 & 1.311 & C4-C5-H27 & 126.2 & 124.3 & $\mathrm{H} 25-\mathrm{C} 3-\mathrm{C} 4-\mathrm{H} 26$ & -69.30 & \\
\hline $\mathrm{N} 8-\mathrm{C} 16$ & 1.385 & 1.393 & C6-C5-H27 & 121.9 & 124.3 & C1-C3-C7-C6 & 140.65 & \\
\hline C9-N10 & 1.388 & 1.374 & C5-C6-C7 & 103.2 & 104.2 & $\mathrm{C} 1-\mathrm{C} 3-\mathrm{C} 7-\mathrm{H} 29$ & 23.72 & \\
\hline C9-H31 & 1.081 & 0.950 & C5-C6-N10 & 113.6 & 111.8 & C1-C3-C7-H30 & -96.00 & \\
\hline N10-C11 & 1.380 & 1.373 & C5-C6-H28 & 110.8 & 109.2 & C4-C3-C7-C6 & 16.61 & \\
\hline C11-N12 & 1.339 & 1.345 & C7-C6-N10 & 114.0 & 113.3 & $\mathrm{C} 4-\mathrm{C} 3-\mathrm{C} 7-\mathrm{H} 29$ & -100.32 & \\
\hline C11-C16 & 1.396 & 1.383 & C7-C6-H28 & 111.0 & 109.2 & C4-C3-C7-H30 & 139.96 & \\
\hline N12-C13 & 1.344 & 1.334 & N10-C6-H28 & 104.4 & 109.2 & H25-C3-C7-C6 & -99.75 & \\
\hline C13-N14 & 1.355 & 1.370 & C3-C7-C6 & 106.2 & 105.9 & $\mathrm{H} 25-\mathrm{C} 3-\mathrm{C} 7-\mathrm{H} 29$ & 143.32 & \\
\hline C13-N21 & 1.374 & 1.355 & C3-C7-H29 & 109.8 & 110.5 & $\mathrm{H} 25-\mathrm{C} 3-\mathrm{C} 7-\mathrm{H} 30$ & 23.60 & \\
\hline N14-C15 & 1.338 & 1.347 & $\mathrm{C} 3-\mathrm{C} 7-\mathrm{H} 30$ & 113.1 & 110.5 & C3-C4-C5-C6 & -0.81 & \\
\hline C15-C16 & 1.414 & 1.412 & C6-C7-H29 & 108.4 & 110.5 & $\mathrm{C} 3-\mathrm{C} 4-\mathrm{C} 5-\mathrm{H} 27$ & -177.54 & \\
\hline C15-N17 & 1.362 & 1.346 & C6-C7-H30 & 112.1 & 110.5 & $\mathrm{H} 26-\mathrm{C} 4-\mathrm{C} 5-\mathrm{C} 6$ & 175.80 & \\
\hline N17-C18 & 1.432 & 1.434 & $\mathrm{H} 29-\mathrm{C} 7-\mathrm{H} 30$ & 107.3 & 108.7 & $\mathrm{H} 26-\mathrm{C} 4-\mathrm{C} 5-\mathrm{H} 27$ & -0.93 & \\
\hline N17-H32 & 1.011 & 0.880 & C9-N8-C16 & 103.9 & - & C4-C5-C6-C7 & 11.47 & \\
\hline C18-C19 & 1.507 & 1.503 & N8-C9-N10 & 113.8 & - & C4-C5-C6-N10 & 135.48 & \\
\hline $\mathrm{C} 18-\mathrm{C} 20$ & 1.506 & 1.493 & N8-C9-H31 & 125.3 & 122.9 & C4-C5-C6-H28 & -107.38 & \\
\hline C18- H33 & 1.089 & 1.000 & N10-C9-H31 & 120.9 & 122.9 & H27-C5-C6-C7 & -171.64 & \\
\hline C19-C20 & 1.509 & 1.511 & C6-N10-C9 & 128.2 & 129.2 & H27-C5-C6-N10 & -47.63 & \\
\hline C19-H34 & 1.084 & 0.990 & C6-N10-C11 & 125.9 & 125.0 & $\mathrm{H} 27-\mathrm{C} 5-\mathrm{C} 6-\mathrm{H} 28$ & 69.51 & \\
\hline C19-H35 & 1.085 & 0.990 & C9-N10-C11 & 105.9 & 105.8 & C5-C6-C7-C3 & -17.12 & \\
\hline $\mathrm{C} 20-\mathrm{H} 36$ & 1.085 & 0.990 & N10-C11-N12 & 127.8 & - & C5-C6-C7-H29 & 100.73 & \\
\hline $\mathrm{C} 20-\mathrm{H} 37$ & 1.086 & 0.990 & N10-C11-C16 & 105.1 & 105.6 & C5-C6-C7-H30 & -141.05 & \\
\hline N21-H38 & 1.008 & 0.879 & N12-C16-C11 & 127.1 & 127.6 & N10-C6-C7-C3 & -140.86 & \\
\hline \multirow[t]{15}{*}{ N21-H39 } & 1.008 & 0.880 & C11-N12-C13 & 111.3 & 111.4 & N10-C6-C7-H29 & -23.01 & \\
\hline & & & N12-C13-N14 & 128.3 & 127.5 & N10-C6-C7-H30 & 95.21 & \\
\hline & & & N12-C13-N21 & 116.5 & 117.2 & $\mathrm{H} 28-\mathrm{C} 6-\mathrm{C} 7-\mathrm{C} 3$ & 101.59 & \\
\hline & & & N14-C13-N21 & 115.2 & 115.2 & $\mathrm{H} 28-\mathrm{C} 6-\mathrm{C} 7-\mathrm{H} 29$ & -140.56 & \\
\hline & & & C13-N14-C15 & 118.2 & 118.7 & $\mathrm{H} 28-\mathrm{C} 6-\mathrm{C} 7-\mathrm{H} 30$ & -22.33 & \\
\hline & & & N14-C15-C16 & 119.3 & 118.5 & C5-C6-N10-C9 & -44.86 & \\
\hline & & & N14-C15-N17 & 120.0 & 118.9 & C5-C6-N10-C11 & 138.03 & 146.99 \\
\hline & & & C16-C15-N17 & 120.7 & 122.6 & C7-C6-N10-C9 & 73.04 & \\
\hline & & & N8-C16-C11 & 111.4 & 105.7 & C7-C6-N10-C11 & -104.07 & \\
\hline & & & N8-C16-C15 & 132.9 & 132.8 & H28-C6-N10-C9 & -165.68 & \\
\hline & & & C11-C16-C15 & 115.7 & 116.1 & H28-C6-N10-C11 & 17.22 & \\
\hline & & & C15-N17-C18 & 123.9 & 122.4 & C16-N8-C9-N10 & 0.30 & \\
\hline & & & C15-N17-H32 & 115.2 & 118.8 & C16-N8-C9-H31 & 179.60 & \\
\hline & & & C18-N17-H32 & 118.6 & 118.8 & C9-N8-C16-C11 & -0.25 & \\
\hline & & & N17-C18-C19 & 119.2 & 117.7 & C9-N8-C16-C15 & -179.16 & \\
\hline
\end{tabular}




\begin{tabular}{|c|c|c|c|c|c|c|c|c|}
\hline & & & N19-C18-C20 & 117.3 & 117.6 & N8-C9-N10-C6 & -177.81 & \\
\hline & & & N17-C18-H33 & 115.3 & 116.5 & N8-C9-N10-C11 & -0.25 & \\
\hline & & & C19-C18-H33 & 116.6 & 116.5 & H31-C9-N10-C6 & 2.86 & \\
\hline & & & $\mathrm{C} 20-\mathrm{C} 18-\mathrm{H} 33$ & 117.5 & 116.5 & H31-C9-N10-C11 & -179.57 & \\
\hline & & & C18-C19-H34 & 116.8 & 117.8 & C6-N10-C11-N12 & -2.71 & \\
\hline & & & C18-C19-H35 & 117.7 & 117.8 & C6-N10-C11-C16 & 177.71 & 179.02 \\
\hline & & & C20-C19-H34 & 117.8 & 117.8 & C9-N10-C11-N12 & 179.65 & \\
\hline & & & C20-C19-H35 & 118.8 & 117.8 & C9-N10-C11-C16 & 0.07 & \\
\hline & & & H34-C19-H35 & 114.9 & 115.0 & N10-C11-N12-C13 & -179.41 & \\
\hline & & & $\mathrm{C} 18-\mathrm{C} 20-\mathrm{H} 36$ & 117.9 & 117.8 & C16-C11-N12-C13 & 0.09 & \\
\hline & & & C18-C20-H37 & 117.4 & 117.8 & N10-C11-C16-N8 & 0.11 & \\
\hline & & & C19-C20-H36 & 119.0 & 117.8 & N10-C11-C16-C15 & 179.22 & \\
\hline & & & C19-C20-H37 & 117.5 & 117.8 & N12-C11-C16-N8 & -179.48 & \\
\hline & & & H36-C20-H37 & 114.4 & 114.9 & N12-C11-C16-C15 & -0.37 & \\
\hline & & & C13-N21-H38 & 115.6 & 120.0 & C11-N12-C13-N14 & 0.72 & \\
\hline & & & C13-N21-H39 & 115.9 & 120.0 & C11-N12-C13-N21 & -177.71 & \\
\hline & & & H38-N21-H39 & 117.2 & 120.0 & N12-C13-N14-C15 & -1.18 & \\
\hline & & & & & & N21-C13-N14-C15 & 177.27 & \\
\hline & & & & & & N12-C13-N21-H38 & -162.32 & \\
\hline & & & & & & N12-C13-N21-H39 & -19.49 & \\
\hline & & & & & & N14-C13-N21-H38 & 19.05 & \\
\hline & & & & & & N14-C13-N21-H39 & 161.87 & \\
\hline & & & & & & C13-N14-C15-C16 & 0.76 & \\
\hline & & & & & & C13-N14-C15-N17 & -178.99 & \\
\hline & & & & & & N14-C15-C16-N8 & 178.78 & \\
\hline & & & & & & N14-C15-C16-C11 & -0.09 & \\
\hline & & & & & & N17-C15-C16-N8 & -1.47 & \\
\hline & & & & & & N17-C15-C16-C11 & 179.66 & \\
\hline & & & & & & N14-C15-N17-C18 & -7.80 & \\
\hline & & & & & & N14-C15-N17-H32 & -170.52 & \\
\hline & & & & & & C16-C15-N17-C18 & 172.45 & \\
\hline & & & & & & C16-C15-N17-H32 & 9.72 & \\
\hline & & & & & & C15-N17-C18-C19 & 82.69 & \\
\hline & & & & & & C15-N17-C18-C20 & 151.93 & 142.2 \\
\hline & & & & & & C15-N17-C18-H33 & -63.34 & \\
\hline & & & & & & H32-N17-C18-C19 & -115.13 & \\
\hline & & & & & & H32-N17-C18-C20 & -45.89 & \\
\hline & & & & & & H32-N17-C18-H33 & 98.84 & \\
\hline & & & & & & N17-C18-C19-H34 & -1.64 & \\
\hline & & & & & & N17-C18-C19-H35 & -144.50 & \\
\hline & & & & & & H33-C18-C19-H34 & 143.95 & \\
\hline & & & & & & H33-C18-C19-H35 & 1.09 & \\
\hline & & & & & & N17-C18-C20-H36 & 141.20 & \\
\hline & & & & & & N17-C18-C20-H37 & -2.09 & \\
\hline & & & & & & H33-C18-C20-H36 & -2.75 & \\
\hline & & & & & & $\mathrm{H} 33-\mathrm{C} 18-\mathrm{C} 20-\mathrm{H} 37$ & -146.04 & \\
\hline & & & & & & $\mathrm{H} 34-\mathrm{C} 19-\mathrm{C} 20-\mathrm{H} 36$ & -146.28 & \\
\hline & & & & & & H34-C19-C20-H37 & -0.91 & \\
\hline & & & & & & $\mathrm{H} 35-\mathrm{C} 19-\mathrm{C} 20-\mathrm{H} 36$ & 0.18 & \\
\hline & & & & & & H35-C19-C20-H37 & 145.55 & \\
\hline
\end{tabular}

$\mathrm{N}-\mathrm{C}-\mathrm{N}$ bond angle for purine ring observed at $113.9^{\circ}$ [19]. The purine ring bond angle N12$\mathrm{C} 13-\mathrm{N} 14=128.3^{\circ}$ by DFT, $127.5^{\circ}$ by XRD, this bond angle is good agreement with literature value [19]. The bond angle of cyclopendane ring has been calculated at $\mathrm{C} 4-\mathrm{C} 3-\mathrm{C} 7=102.8^{\circ}$ by DFT and $103.2^{\circ}$ by XRD shows good correlation between calculated and experimental values. The cyclopropyl group C-C-C bond angle has been calculated at $\mathrm{N} 19-\mathrm{C} 18-\mathrm{C} 20=117.3^{\circ}$ by DFT method. The dihedral angle between purine and cyclopropyl ring has been observed at C15-N17-C18$\mathrm{C} 20=151.93^{\circ}$ (DFT), $142.2^{\circ}$ (XRD), similarly dihedral angle between purine and cyclopendane ring is $\mathrm{C} 6-\mathrm{N} 10-\mathrm{C} 11-\mathrm{C} 16=177.71^{\circ}$ (DFT), $179.02^{\circ}$ (XRD) and $\mathrm{C} 5-\mathrm{C} 6-\mathrm{N} 10-\mathrm{C} 11=138.03^{\circ}(\mathrm{DFT})$, $146.99^{\circ}$ (DFT) respectively. 


\subsection{Vibrational assignments}

The detailed vibrational assignments (characterized by PED) of fundamental modes of Abacavir along with the calculated IR and Raman intensities are reported in Table 2. Theoretical and corresponding scaled (SQM) frequencies were calculated for title compound at B3LYP/6$31 \mathrm{G}(\mathrm{d}, \mathrm{p})$ level have been collected along with IR intensities and Raman scattering activities. Theoretically calculated wavenumbers are usually larger than the experimental values, due to the exclusion of scaled frequencies, incomplete incorporation of electron correlation and the use of finite basis set. The over estimations are mostly systematic and can be corrected by following an empirical scaling procedure over the obtained theoretical frequencies fit to the corresponding experimental frequencies. In this study, selective scaling procedures were applied. For the B3LYP/6-31G(d,p) level, the scale factors of 0.9608 reported in reference [20] respectively.

\subsubsection{Purine Ring vibrations}

The C-C stretching vibrations of the purine ring observed in the Spectral region over 1650$1000 \mathrm{~cm}^{-1}$ [21]. In our present investigation $\mathrm{C}-\mathrm{C}$ stretching vibrations observed at $1606 \mathrm{~cm}^{-1}$ in FTIR and $1607 \mathrm{~cm}^{-1}$ in FT-Raman spectrum and calculated at $1595 \mathrm{~cm}^{-1}$ and $1569 \mathrm{~cm}^{-1}$ by DFT method. The hetero aromatic structure shows the presence of $\mathrm{C}-\mathrm{H}$ stretching vibrations in the region $3100-3000 \mathrm{~cm}^{-1}$, which is the characteristic region for the ready identification of $\mathrm{CH}$ stretching vibrations [22]. For title compound this vibration observed at $3196 \mathrm{~cm}^{-1}$ in FT-IR spectrum and $3131 \mathrm{~cm}^{-1}$ by DFT method this is a pure mode, the contribution of PED for this mode is $100 \%$. Silverstein et al. [23] assigned C-N, C=N stretching absorption in the region $1382-1266 \mathrm{~cm}^{-1}$ for aromatic amines. In our work the purine ring $\mathrm{C}-\mathrm{N}$ stretching vibration observed at $1500 \mathrm{~cm}^{-1}$ in FTIR spectrum and 1500, 1307 and $1168 \mathrm{~cm}^{-1}$ in FT-Raman spectrum. The theoretically predicted wave numbers at $1585,1499,1471,1419,1370,1292$ and $1289 \mathrm{~cm}^{-1}$ by DFT method gives the C-N stretching vibrations. The N-C-N and C-C-N inplane bending vibrations of the purine ring observed at $828 \mathrm{~cm}^{-1}$ in IR spectrum and $362 \mathrm{~cm}^{-1}$ in FT-Raman spectrum, the calculated wavenumbers at $1005,824,364 \mathrm{~cm}^{-1}$ and $726,433,427 \mathrm{~cm}^{-1}$ by DFT method gives the N-C-N and C-C-N inplane bending vibrations respectively. Mode no's 107 and 109 gives the CCCN out of plane bending vibration of the purine ring.

\subsubsection{Amino group vibration}

According to Socrates [23] the stretching of amino group appeared around 3500-3000 $\mathrm{cm}^{-1}$ in absorption spectra. For title molecule the wavenumbers $3465 \mathrm{~cm}^{-1}$ and $3592 \mathrm{~cm}^{-1}$ calculated by DFT calculations gives the $\mathrm{N}-\mathrm{H}$ symmetric and antisymmetric stretching vibrations, no bands observed in FT-IR and FT-Raman spectrums for this vibration. The wavenumber at $1569 \mathrm{~cm}^{-1}$ predicted by DFT method has been identified as $\mathrm{NH}_{2}$ scissoring vibration.

\subsubsection{Cyclopentane ring vibrations}

The C-C stretching vibration of the cyclopentane ring has been computed the scaled wavenumbers at $1029,904,812 \mathrm{~cm}^{-1}$ by DFT method. The FT-IR band at $750,564 \mathrm{~cm}^{-1}$ and FTRaman band at $565 \mathrm{~cm}^{-1}$ are identified as $\mathrm{C}-\mathrm{C}-\mathrm{C}$ inplane bending vibrations of the cyclopentane ring. C-C-C-C out-of plane bending vibrations observed at $442,324 \mathrm{~cm}^{-1}$ by DFT method and $321 \mathrm{~cm}^{-1}$ in FT-Raman band. The C-H stretching vibrations of the cyclopentane ring observed at $2967 \mathrm{~cm}^{-1}$ in FT-IR spectrum and $2904 \mathrm{~cm}^{-1}$ in FT-Raman spectrum. The computed wavenumbers at $3090,3062,3003,2944$ and $2905 \mathrm{~cm}^{-1}$ by DFT method gives the C-C stretching vibrations. The $\mathrm{CH}$ inplane bending vibrations have been identified at $1258,1098 \mathrm{~cm}^{-1}$ in FT-IR and 1250, 1096 $\mathrm{cm}^{-1}$ in FT-Raman spectrum. The mode no's 40, 41, 47, 50 and 51 have been identified as $\mathrm{CH}$ inplane bending vibrations. The computed wavenumbers at 1029, 904, 763 and $753 \mathrm{~cm}^{-1}$ gives the $\mathrm{CCCH}$ torsional vibrations respectively. 

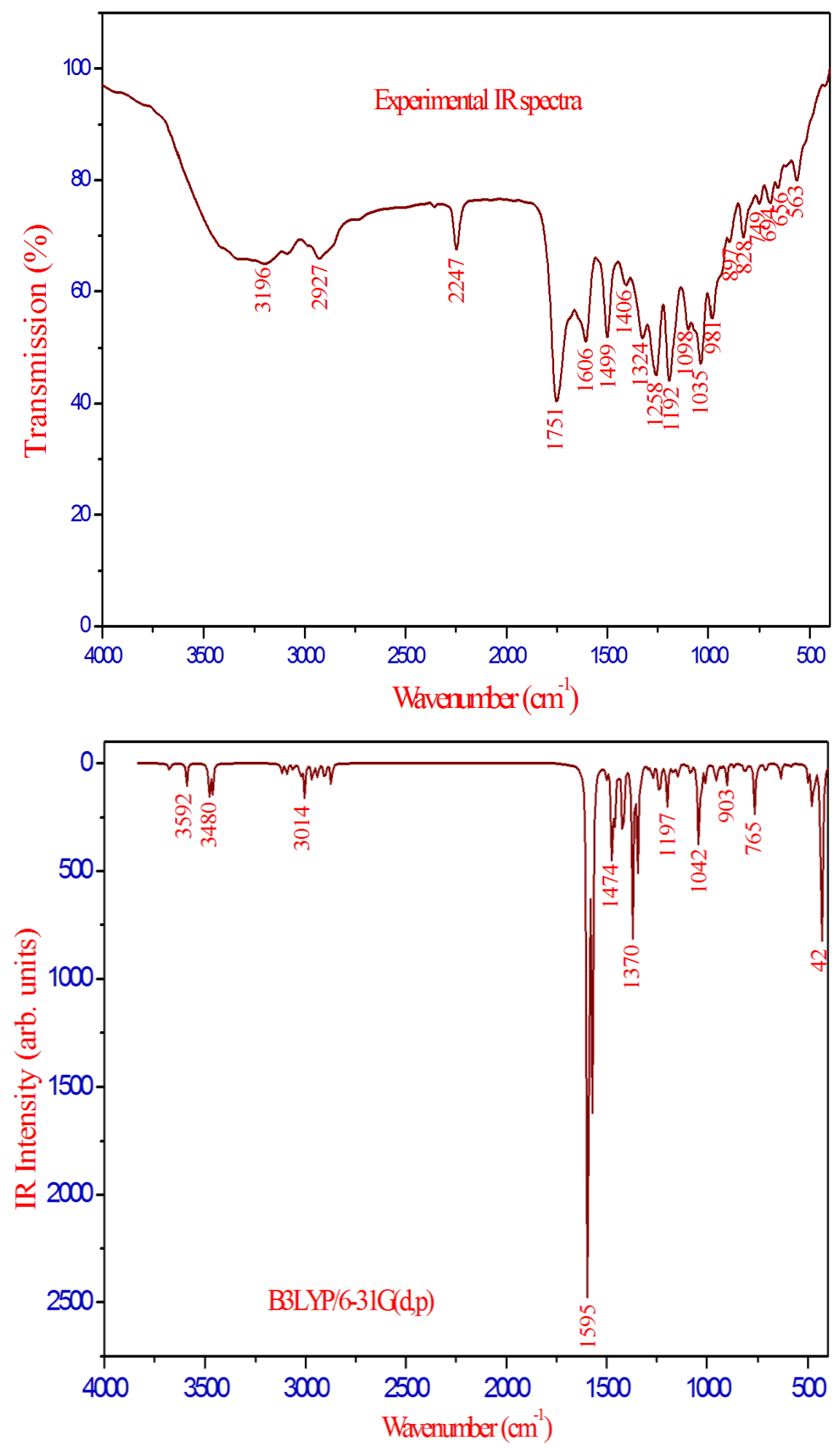

Figure 2. Comparison of experimental and theoretical B3LYP/6-31G(d,p) FT-IR spectrum for Abacavir. 

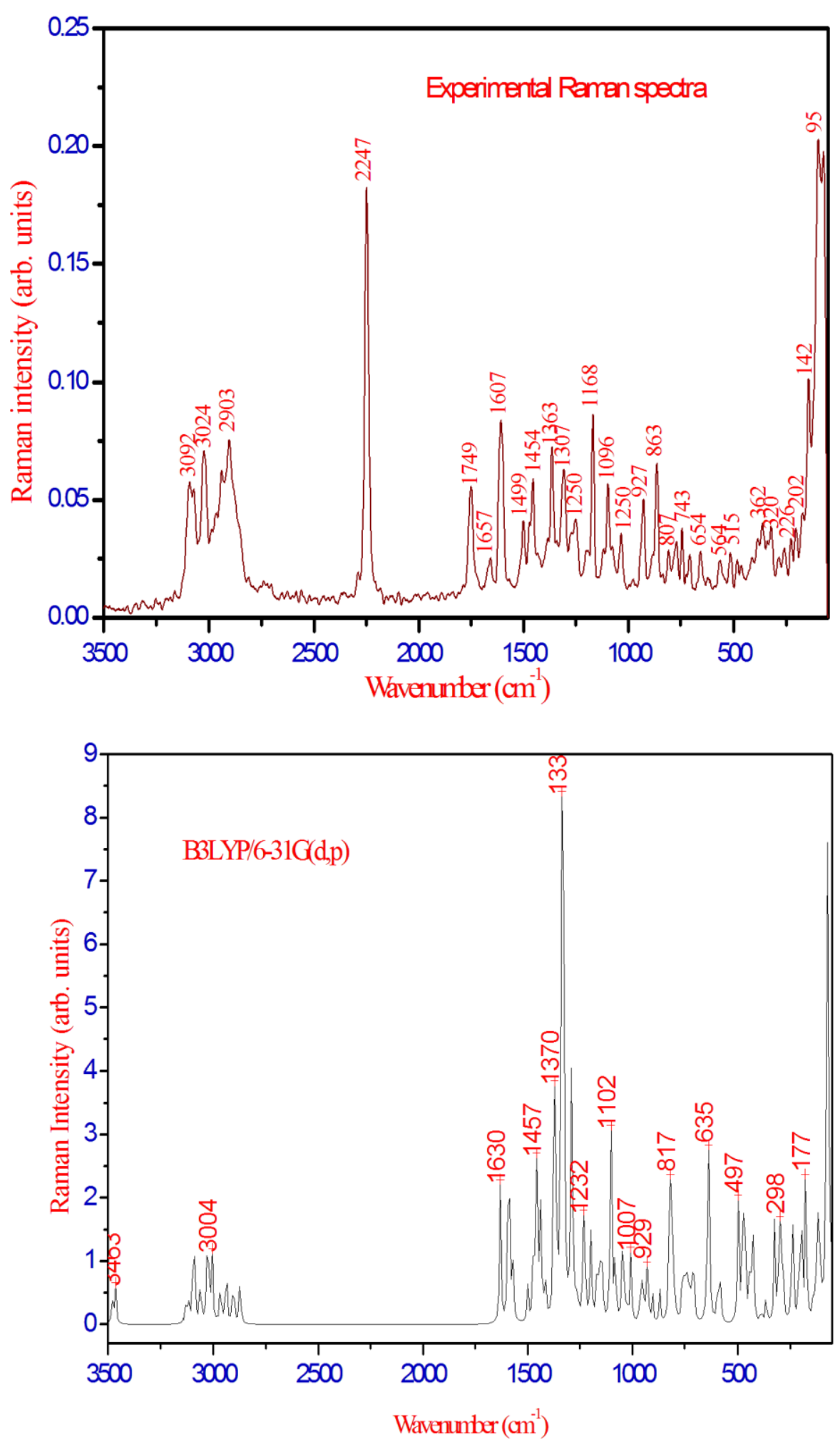

Figure 3. Comparison of experimental and theoretical B3LYP/6-31G(d,p) FT-Raman spectrum for Abacavir. 
Table 2. Comparison of the experimental and calculated vibrational spectra and proposed assignments of Abacavir.

\begin{tabular}{|c|c|c|c|c|c|c|c|}
\hline \multirow{3}{*}{$\begin{array}{l}\text { Mode } \\
\text { No }\end{array}$} & \multicolumn{2}{|c|}{$\begin{array}{l}\text { Experimental wave } \\
\text { numbers } / \mathrm{cm}^{-1}\end{array}$} & \multicolumn{4}{|c|}{ Theoretical wave numbers $/ \mathrm{cm}^{-1}$} & \multirow{3}{*}{$\begin{array}{c}\text { Vibrational assignments with PED } \\
(\geq 10 \%)\end{array}$} \\
\hline & \multirow{2}{*}{ FT-IR } & \multirow{2}{*}{$\begin{array}{c}\text { FT- } \\
\text { Raman }\end{array}$} & \multicolumn{4}{|c|}{ B3LYP/6-31G(d,p) } & \\
\hline & & & Unscaled & scaled & $\mathbf{I}_{I R}{ }^{\mathbf{a}}$ & $\mathbf{I}_{\mathbf{R a}}{ }^{\mathbf{b}}$ & \\
\hline 1 & & & 3825 & 3676 & 13.49 & 1.89 & $v_{\mathrm{O} 2 \mathrm{H} 24}$ \\
\hline 2 & & & 3739 & 3592 & 30.52 & 2.39 & $V_{\text {asymN21H38(100) }}$ \\
\hline 3 & & & 3623 & 3481 & 44.19 & 2.94 & $v_{\mathrm{N} 17 \mathrm{H} 32(100)}$ \\
\hline 4 & & & 3606 & 3465 & 53.57 & 7.21 & $v_{\text {symN21H38(100) }}$ \\
\hline 5 & 3196 & & 3259 & 3131 & 0.28 & 3.54 & $v_{\mathrm{C} 9 \mathrm{H} 31(100)}$ \\
\hline 6 & & & 3246 & 3119 & 16.21 & 2.93 & $V_{\text {asymC19H34(88) }}$ \\
\hline 7 & & & 3226 & 3100 & 5.96 & 4.98 & $V_{\text {asymC20H } 36(89)}$ \\
\hline 8 & & 3092 & 3216 & 3090 & 13.14 & 7.31 & $v_{\mathrm{C} 5 \mathrm{H} 27(79)}$ \\
\hline 9 & & & 3187 & 3062 & 11.03 & 6.17 & $v_{\mathrm{C} 4 \mathrm{H} 26(80)}$ \\
\hline 10 & & & 3156 & 3032 & 8.44 & 8.06 & $V_{\text {symC19H34(95) }}$ \\
\hline 11 & & 3024 & 3143 & 3020 & 15.78 & 6.96 & $v_{\text {symC2 } 20 \mathrm{H} 36(95)}$ \\
\hline 12 & & & 3128 & 3005 & 31.06 & 5.68 & $v_{\mathrm{C} 18 \mathrm{H} 33(98)}$ \\
\hline 13 & & & 3126 & 3003 & 17.67 & 3.21 & $v_{\mathrm{C} 7 \mathrm{H} 30(84)}$ \\
\hline 14 & & & 3088 & 2967 & 34.12 & 5.77 & $V_{\text {asymC1H23(93) }}$ \\
\hline 15 & & & 3064 & 2944 & 14.43 & 2.01 & $v_{\mathrm{C} 6 \mathrm{H} 28(77)}$ \\
\hline 16 & 2927 & & 3057 & 2937 & 8.39 & 5.19 & $v_{\mathrm{C} 7 \mathrm{H} 29(69)}$ \\
\hline 17 & & 2904 & 3024 & 2905 & 33.13 & 6.56 & $v_{\mathrm{C} 3 \mathrm{H} 25(94)}$ \\
\hline 18 & & & 2990 & 2872 & 36.85 & 5.66 & $v_{\mathrm{symC1H} 22(91)}$ \\
\hline 19 & & 1658 & 1697 & 1631 & 1.45 & 12.73 & $v_{\mathrm{C} 4 \mathrm{C} 5(75)}$ \\
\hline 20 & 1606 & 1607 & 1660 & 1595 & 669.36 & 8.20 & $v_{\mathrm{C} 15 \mathrm{C} 16(20)+} \delta_{\mathrm{H} 32 \mathrm{~N} 17 \mathrm{C} 15(14)}$ \\
\hline 21 & & & 1649 & 1585 & 305.64 & 10.16 & $v_{\mathrm{N} 12 \mathrm{C} 11(24)+} \delta_{\mathrm{C} 16 \mathrm{C} 11 \mathrm{~N} 10(24)}$ \\
\hline 22 & & & 1633 & 1569 & 440.85 & 4.40 & $\delta_{\mathrm{H} 38 \mathrm{~N} 21 \mathrm{H} 39(46)+} v_{\mathrm{C} 11 \mathrm{C} 16(30)}$ \\
\hline 23 & 1500 & 1500 & 1560 & 1499 & 15.15 & 2.98 & $v_{\mathrm{N} 8 \mathrm{C} 9(44)+} \delta_{\mathrm{H} 31 \mathrm{C} 9 \mathrm{~N} 8(11)}$ \\
\hline 24 & & & 1531 & 1471 & 180.90 & 6.29 & $v_{\mathrm{N} 8 \mathrm{C} 9(56)}$ \\
\hline 25 & & 1455 & 1518 & 1459 & 2.74 & 3.30 & 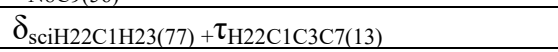 \\
\hline 26 & & & 1517 & 1458 & 76.64 & 10.98 & $\tau_{\mathrm{H} 22 \mathrm{C} 1 \mathrm{C} 3 \mathrm{C} 7(13)}$ \\
\hline 27 & & & 1504 & 1445 & 3.89 & 2.76 & $\delta_{\mathrm{H} 29 \mathrm{C} 7 \mathrm{H} 30(89)}$ \\
\hline 28 & & & 1496 & 1437 & 8.79 & 10.57 & $v_{\mathrm{N} 12 \mathrm{C} 11(11)+} \delta_{\mathrm{C} 11 \mathrm{C} 16 \mathrm{~N} 8(14)}$ \\
\hline 29 & & & 1477 & 1419 & 104.66 & 0.63 & $v_{\mathrm{N} 12 \mathrm{C} 11(27)+} \delta_{\mathrm{H} 32 \mathrm{~N} 17 \mathrm{C} 15(18)+} \delta_{\mathrm{sciH} 36 \mathrm{C} 20 \mathrm{H} 37(51)}$ \\
\hline 30 & 1407 & & 1473 & 1415 & 54.46 & 3.07 & $\delta_{\text {sciH34C19H35(71) }}$ \\
\hline 31 & & & 1434 & 1378 & 3.23 & 7.11 & $\delta_{\mathrm{H} 28 \mathrm{C} 6 \mathrm{~N} 10(45)}$ \\
\hline 32 & & & 1426 & 1370 & 222.36 & 15.37 & $v_{\mathrm{N} 10 \mathrm{C} 11(21)+} \delta_{\mathrm{H} 28 \mathrm{C} 6 \mathrm{~N} 10(10)}$ \\
\hline 33 & & 1363 & 1419 & 1364 & 23.49 & 3.78 & $\delta_{\text {wagH22C1H23(12) }} \gamma_{\mathrm{H} 22 \mathrm{C} 1 \mathrm{C} 3 \mathrm{C} 7(47)}$ \\
\hline 34 & & & 1401 & 1346 & 155.76 & 1.15 & $\delta_{\mathrm{H} 33 \mathrm{C} 18 \mathrm{~N} 17(44)}$ \\
\hline 35 & & & 1397 & 1343 & 9.86 & 8.42 & $\delta_{\mathrm{H} 22 \mathrm{C} 1 \mathrm{OO} 2(46)}$ \\
\hline 36 & & & 1392 & 1337 & 6.46 & 12.73 & $\delta_{\mathrm{H} 26 \mathrm{C} 4 \mathrm{C} 5(35)}$ \\
\hline 37 & 1325 & & 1385 & 1331 & 31.90 & 64.03 & $v_{\mathrm{C} 15 \mathrm{C} 16(10)}$ \\
\hline 38 & & 1307 & 1345 & 1292 & 3.71 & 9.66 & $v_{\mathrm{N} 8 \mathrm{C} 16(22)}$ \\
\hline 39 & & & 1341 & 1289 & 1.85 & 17.25 & $v_{\mathrm{N} 8 \mathrm{C} 16(11)}+\delta_{\mathrm{H} 25 \mathrm{C} 3 \mathrm{C} 4(19)+}+\gamma_{\mathrm{C} 6 \mathrm{C} 5 \mathrm{~N} 10 \mathrm{H} 28(12)}$ \\
\hline 40 & & & 1322 & 1270 & 30.28 & 1.65 & $\delta_{\mathrm{H} 25 \mathrm{C} 3 \mathrm{C} 4(42)}$ \\
\hline 41 & 1258 & 1250 & 1313 & 1261 & 1.98 & 1.27 & $\delta_{\mathrm{H} 26 \mathrm{CC} 55(13)+} \gamma_{\mathrm{C} 6 \mathrm{C} 5 \mathrm{~N} 10 \mathrm{H} 28(49)}$ \\
\hline 42 & & & 1288 & 1238 & 44.56 & 2.60 & $\tau_{\mathrm{H} 25 \mathrm{C} 3 \mathrm{C} 4 \mathrm{C} 5(37)}$ \\
\hline 43 & & & 1279 & 1229 & 25.37 & 10.75 & $v_{\mathrm{N} 17 \mathrm{C} 18(10)+} \tau_{\mathrm{H} 25 \mathrm{C} 3 \mathrm{C} 4 \mathrm{C} 5(17)}$ \\
\hline 44 & 1192 & & 1248 & 1199 & 63.94 & 7.43 & $v_{\mathrm{C} 18 \mathrm{C} 19(11)+} \delta_{\mathrm{H} 33 \mathrm{C} 18 \mathrm{~N} 17(16)}$ \\
\hline 45 & & & 1235 & 1187 & 9.53 & 0.61 & $\delta_{\mathrm{H} 31 \mathrm{C} 9 \mathrm{~N} 8(49)}$ \\
\hline 46 & & 1168 & 1218 & 1170 & 8.11 & 3.05 & $v_{\mathrm{N} 10 \mathrm{C} 9(50)}$ \\
\hline 47 & & & 1207 & 1160 & 8.58 & 3.07 & $\delta_{\mathrm{H} 25 \mathrm{C} 3 \mathrm{C} 4(21)}$ \\
\hline 48 & & & 1199 & 1152 & 0.22 & 2.73 & $\delta_{\mathrm{H} 34 \mathrm{C} 19 \mathrm{C} 20(51)+} \gamma_{\mathrm{H} 34 \mathrm{C} 19 \mathrm{C} 20 \mathrm{C} 18(15)}$ \\
\hline 49 & & & 1197 & 1150 & 2.66 & 1.45 & $\delta_{\mathrm{H} 34 \mathrm{C} 19 \mathrm{C} 20(32)}$ \\
\hline 50 & & & 1189 & 1143 & 21.32 & 4.13 & $\delta_{\mathrm{H} 25 \mathrm{C} 3 \mathrm{C} 4(13)}$ \\
\hline 51 & 1098 & 1096 & 1148 & 1103 & 1.44 & 15.79 & $\delta_{\mathrm{H} 26 \mathrm{C} 4 \mathrm{C} 5(69)}$ \\
\hline 52 & & & 1130 & 1085 & 9.21 & 3.21 & $\delta_{\mathrm{H} 32 \mathrm{~N} 21 \mathrm{C} 13(12)}$ \\
\hline 53 & & & 1126 & 1082 & 1.74 & 1.33 & $\tau_{\mathrm{H} 33 \mathrm{C} 18 \mathrm{~N} 17 \mathrm{C} 15(65)}$ \\
\hline 54 & & & 1119 & 1075 & 5.90 & 1.02 & $v_{\mathrm{C} 1 \mathrm{C} 3(20)}$ \\
\hline
\end{tabular}




\begin{tabular}{|c|c|c|c|c|c|c|c|}
\hline 55 & & & 1093 & 1050 & 16.61 & 4.49 & $v_{\mathrm{N} 12 \mathrm{C} 13(23)+} \delta_{\mathrm{H} 38 \mathrm{~N} 21 \mathrm{C} 13(28)}$ \\
\hline 56 & & & 1083 & 1041 & 105.37 & 3.28 & $v_{\mathrm{O} 2 \mathrm{Cl}(65)}$ \\
\hline 57 & 1036 & 1034 & 1079 & 1036 & 2.60 & 0.49 & $\tau_{\mathrm{H} 36 \mathrm{C} 20 \mathrm{C} 19 \mathrm{C} 18(80)}$ \\
\hline 58 & & & 1071 & 1029 & 49.38 & 0.74 & $v_{\mathrm{C} 3 \mathrm{C} 7(17)+} \delta_{\mathrm{H} 24 \mathrm{O} 2 \mathrm{C} 1(12)}+\tau_{\mathrm{H} 22 \mathrm{C} 1 \mathrm{C} 3 \mathrm{C} 7(13)}$ \\
\hline 59 & & & 1063 & 1022 & 5.09 & 0.64 & $v_{\mathrm{N} 14 \mathrm{C} 15(17)+} \tau_{\mathrm{H} 34 \mathrm{C} 19 \mathrm{C} 20 \mathrm{C} 18(64)}$ \\
\hline 60 & & & 1049 & 1008 & 18.18 & 3.56 & $v_{\mathrm{C} 5 \mathrm{C} 6(19)+} \delta_{\mathrm{C} 5 \mathrm{C} 6 \mathrm{~N} 10(10)+} \gamma_{\mathrm{C} 7 \mathrm{C} 3 \mathrm{C} 6 \mathrm{H} 29(14)}$ \\
\hline 61 & & & 1046 & 1005 & 7.19 & 2.35 & $v_{\mathrm{N} 12 \mathrm{C} 13(10)+} \delta_{\mathrm{N} 8 \mathrm{C} 9 \mathrm{~N} 10(10)+} \tau_{\mathrm{H} 34 \mathrm{C} 19 \mathrm{C} 20 \mathrm{C} 18(13)}$ \\
\hline 62 & 981 & & 1005 & 966 & 8.73 & 1.68 & $v_{\mathrm{C} 3 \mathrm{C} 7(23)}$ \\
\hline 63 & & & 992 & 953 & 29.08 & 3.58 & $v_{\mathrm{C} 19 \mathrm{C} 20(19)+} \tau_{\mathrm{H} 34 \mathrm{C} 19 \mathrm{C} 20 \mathrm{C} 18(18)}$ \\
\hline 64 & & & 980 & 942 & 1.54 & 1.47 & $\tau_{\mathrm{H} 26 \mathrm{C} 4 \mathrm{C} 5 \mathrm{H} 27(83)}$ \\
\hline 65 & & 927 & 965 & 927 & 4.89 & 4.73 & $v_{\mathrm{C} 18 \mathrm{C} 20(31)+} \tau_{\mathrm{H} 33 \mathrm{C} 18 \mathrm{~N} 17 \mathrm{C} 15(12)}$ \\
\hline 66 & 897 & & 941 & 904 & 31.44 & 2.08 & $v_{\mathrm{C} 5 \mathrm{C} 6(12)+} \tau_{\mathrm{H} 22 \mathrm{C} 1 \mathrm{C} 3 \mathrm{C} 7(31)}$ \\
\hline 67 & & 863 & 904 & 869 & 4.21 & 2.25 & $v_{\mathrm{C} 6 \mathrm{CC} 7(12)+}+\tau_{\mathrm{H} 22 \mathrm{C} 1 \mathrm{C3} 37(15)+} \gamma_{\mathrm{C} 7 \mathrm{C} 3 \mathrm{C} 6 \mathrm{H} 29(21)}$ \\
\hline 68 & 828 & & 858 & 824 & 1.12 & 5.78 & $v_{\mathrm{C} 18 \mathrm{C} 19(11)+} \delta_{\mathrm{N} 12 \mathrm{C} 13 \mathrm{~N} 14(13)}$ \\
\hline 69 & & & 849 & 815 & 5.49 & 8.00 & $v_{\mathrm{C} 18 \mathrm{C} 19(27)}$ \\
\hline 70 & & & 845 & 812 & 9.05 & 3.22 & $v_{\mathrm{C} 6 \mathrm{C} 7(27)}$ \\
\hline 71 & & 807 & 838 & 805 & 4.86 & 4.97 & $v_{\mathrm{C} 19 \mathrm{C} 20(19)+} \tau_{\mathrm{H} 33 \mathrm{C} 18 \mathrm{~N} 17 \mathrm{C} 15(45)}$ \\
\hline 72 & & & 826 & 793 & 1.04 & 0.51 & $\tau_{\mathrm{H} 31 \mathrm{C} 9 \mathrm{~N} 8 \mathrm{C} 16(80)}$ \\
\hline 73 & & 769 & 797 & 765 & 33.96 & 1.07 & $\tau_{\mathrm{C} 11 \mathrm{~N} 10 \mathrm{C} 9 \mathrm{~N} 8(15)}+\gamma_{\mathrm{N} 21 \mathrm{~N} 12 \mathrm{~N} 14 \mathrm{C} 13(12)}$ \\
\hline 74 & & & 794 & 763 & 42.92 & 1.31 & $\tau_{\mathrm{H} 26 \mathrm{C} 4 \mathrm{C} 5 \mathrm{C} 6(15)}$ \\
\hline 75 & 750 & & 783 & 753 & 11.42 & 4.19 & $\delta_{\mathrm{C} 4 \mathrm{CSC} 6(21)+} \tau_{\mathrm{H} 26 \mathrm{C} 4 \mathrm{CSC} 6(40)}$ \\
\hline 76 & & 743 & 772 & 742 & 0.94 & 4.23 & $\delta_{\mathrm{H} 34 \mathrm{C} 19 \mathrm{C} 20(79)}$ \\
\hline 77 & & & 756 & 726 & 1.07 & 3.90 & $\delta_{\mathrm{C} 11 \mathrm{C1} 6 \mathrm{~N} 8(11)}$ \\
\hline 78 & & & 741 & 712 & 5.59 & 2.06 & $\delta_{\mathrm{C} 3 \mathrm{C} 4 \mathrm{C} 5(15)+} \tau_{\mathrm{C} 11 \mathrm{~N} 10 \mathrm{C} 9 \mathrm{~N} 8(13)+} \gamma_{\mathrm{N} 21 \mathrm{~N} 12 \mathrm{~N} 14 \mathrm{Cl} 1325)}$ \\
\hline 79 & 695 & 707 & 736 & 707 & 7.93 & 3.62 & $\delta_{\mathrm{C} 3 \mathrm{C} 4 \mathrm{C} 5(14)+} \tau_{\mathrm{C} 11 \mathrm{~N} 10 \mathrm{C} 9 \mathrm{~N} 8(12)+} \gamma_{\mathrm{N} 21 \mathrm{~N} 12 \mathrm{~N} 14 \mathrm{Cl} 1326)}$ \\
\hline 80 & 656 & 655 & 681 & 654 & 0.25 & 0.27 & $\gamma_{\mathrm{N} 12 \mathrm{C} 16 \mathrm{~N} 10 \mathrm{C} 1175)}$ \\
\hline 81 & & & 664 & 638 & 17.13 & 14.77 & $\delta_{\mathrm{N} 10 \mathrm{C} 11 \mathrm{~N} 12(19)}$ \\
\hline 82 & & & 658 & 632 & 13.60 & 2.05 & $\tau_{\mathrm{C} 11 \mathrm{~N} 10 \mathrm{C} 9 \mathrm{~N} 8(69)}$ \\
\hline 83 & & & 620 & 596 & 3.64 & 3.42 & $\delta_{\mathrm{N} 12 \mathrm{C} 13 \mathrm{~N} 14(24)}$ \\
\hline 84 & 564 & 565 & 607 & 583 & 3.79 & 2.33 & $\delta_{\mathrm{C} 4 \mathrm{C5C} 6(13)}$ \\
\hline 85 & & 515 & 518 & 497 & 21.82 & 7.91 & $\delta_{\mathrm{C} 11 \mathrm{~N} 12 \mathrm{C} 13(25)+} \tau_{\mathrm{H} 32 \mathrm{~N} 17 \mathrm{C} 15 \mathrm{C} 16(10)}$ \\
\hline 86 & & 483 & 496 & 477 & 82.13 & 6.22 & $\tau_{\mathrm{H} 32 \mathrm{~N} 17 \mathrm{C} 15 \mathrm{C} 16(73)}$ \\
\hline 87 & & & 488 & 469 & 8.87 & 4.91 & $\delta_{\mathrm{ClC} 3 \mathrm{C} 4(14)+} \tau_{\mathrm{C} 4 \mathrm{C} 5 \mathrm{C} 6 \mathrm{~N} 10(24)}$ \\
\hline 88 & & & 481 & 463 & 14.91 & 3.36 & $\tau_{\mathrm{H} 38 \mathrm{~N} 21 \mathrm{C} 13 \mathrm{~N} 14(73)}$ \\
\hline 89 & & & 460 & 442 & 18.40 & 4.53 & $\gamma_{\mathrm{C3C4C5C6(11)}}$ \\
\hline 90 & & & 451 & 433 & 105.83 & 1.13 & $\delta_{\mathrm{C} 19 \mathrm{C} 18 \mathrm{~N} 17(22)+} \tau_{\mathrm{H} 38 \mathrm{~N} 21 \mathrm{C} 13 \mathrm{~N} 14(20)}$ \\
\hline 91 & & & 444 & 427 & 205.32 & 4.76 & $\delta_{\mathrm{C} 19 \mathrm{C} 18 \mathrm{~N} 17(14)+} \tau_{\mathrm{H} 38 \mathrm{~N} 21 \mathrm{C} 13 \mathrm{~N} 14(52)}$ \\
\hline 92 & & & 404 & 388 & 9.72 & 0.83 & $\delta_{\mathrm{C} 3 \mathrm{C} 1 \mathrm{O} 2(15)}$ \\
\hline 93 & & 362 & 379 & 364 & 16.42 & 2.02 & $\delta_{\mathrm{N} 12 \mathrm{C} 13 \mathrm{~N} 21(27)}+\gamma_{\mathrm{C} 1 \mathrm{C} 4 \mathrm{C} 7 \mathrm{C} 3(12)}$ \\
\hline 94 & & 321 & 337 & 324 & 11.93 & 6.09 & $\gamma_{\mathrm{ClC4C7C3(37)}}$ \\
\hline 95 & & & 314 & 302 & 5.95 & 4.85 & $\delta_{\mathrm{N} 12 \mathrm{C} 13 \mathrm{~N} 21(11)}+\gamma_{\mathrm{C} 1 \mathrm{C} 4 \mathrm{C} 7 \mathrm{C} 3(31)}$ \\
\hline 96 & & & 307 & 295 & 103.11 & 6.48 & $\gamma_{\mathrm{H} 24 \mathrm{O} 2 \mathrm{ClC} 3(70)}$ \\
\hline 97 & & & 295 & 284 & 29.92 & 3.63 & $\tau_{\mathrm{H} 24 \mathrm{O} 2 \mathrm{ClC} 3(16)+}+\gamma_{\mathrm{C} 1 \mathrm{C} 4 \mathrm{C} 7 \mathrm{C} 3(16)}$ \\
\hline 98 & & 258 & 250 & 240 & 5.14 & 8.17 & $v_{\mathrm{C} 15 \mathrm{C} 16+} \delta_{\mathrm{C} 15 \mathrm{~N} 17 \mathrm{C} 18(12)}$ \\
\hline 99 & & 226 & 234 & 225 & 1.65 & 0.16 & $\tau_{\mathrm{C} 13 \mathrm{~N} 12 \mathrm{C} 11 \mathrm{~N} 10(67)}$ \\
\hline 100 & & & 219 & 210 & 1.65 & 1.41 & $\delta_{\mathrm{C} 1 \mathrm{C} 3 \mathrm{C} 4(44)+} \tau_{\mathrm{C} 4 \mathrm{C} 5 \mathrm{C} 6 \mathrm{~N} 10(11)}$ \\
\hline 101 & & 203 & 206 & 198 & 0.34 & 9.22 & $\delta_{\mathrm{C} 5 \mathrm{C} 6 \mathrm{~N} 10(13)}$ \\
\hline 102 & & & 182 & 175 & 3.03 & 10.31 & $\tau_{\mathrm{C} 11 \mathrm{~N} 12 \mathrm{C} 13 \mathrm{~N} 14(26)}$ \\
\hline 103 & & 143 & 144 & 138 & 2.97 & 2.25 & $\tau_{\mathrm{C} 11 \mathrm{~N} 12 \mathrm{C} 13 \mathrm{~N} 14(51)}$ \\
\hline 104 & & & 124 & 119 & 0.46 & 8.36 & $\delta_{\mathrm{C} 6 \mathrm{~N} 10 \mathrm{C} 9(23)}$ \\
\hline 105 & & & 110 & 105 & 3.83 & 4.04 & $\tau_{\mathrm{C} 4 \mathrm{C} 3 \mathrm{C} 1 \mathrm{O} 2(41)}$ \\
\hline 106 & & 96 & 85 & 82 & 2.20 & 5.32 & $\delta_{\mathrm{N} 14 \mathrm{C} 15 \mathrm{~N} 17(18)}$ \\
\hline 107 & & 72 & 74 & 71 & 2.01 & 35.18 & $\gamma_{\mathrm{C6C9C11N10(55)}}$ \\
\hline 108 & & & 46 & 44 & 0.55 & 12.05 & $\tau_{\mathrm{C} 15 \mathrm{~N} 17 \mathrm{C} 18 \mathrm{C} 19(69)}$ \\
\hline 109 & & & 37 & 35 & 0.52 & 6.52 & $\gamma_{\mathrm{C} 6 \mathrm{C} 9 \mathrm{C} 11 \mathrm{~N} 10(46)}$ \\
\hline 110 & & & 24 & 23 & 0.15 & 54.09 & $\tau_{\mathrm{C} 4 \mathrm{C} 5 \mathrm{C} 6 \mathrm{~N} 10(52)+}+\gamma_{\mathrm{C} 6 \mathrm{C} 9 \mathrm{C} 11 \mathrm{~N} 10(12)}$ \\
\hline 111 & & & 18 & 18 & 0.07 & 100.00 & $\gamma_{\mathrm{C} 6 \mathrm{C} 9 \mathrm{C} 1 \mathrm{~N} 10(80)}$ \\
\hline
\end{tabular}

$\nu$-stretching; $\delta$-in-plane-bending; $\gamma$-out-of-plane bending; $\tau$-torsion; w-weak; s-strong; vs-very strong; vw-very weak; m-medium.

${ }^{\mathrm{a}} \mathrm{I}_{\mathrm{IR}}$-IR Intensity $\left(\mathrm{Kmmol}^{-1}\right)$; ${ }^{\mathrm{b}} \mathrm{I}_{\mathrm{Ra}}$-Raman intensity (Arb units) (intensity normalized to $100 \%$ ) 


\subsubsection{Methylene and $\mathrm{OH}$ group vibrations}

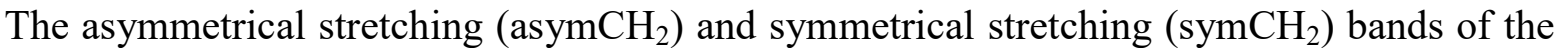
methylene group occur near 2926 and $2853 \mathrm{~cm}^{-1}$, respectively [24]. In our present work the predicted wave numbers at $2967 \mathrm{~cm}^{-1}$ and $2872 \mathrm{~cm}^{-1}$ are identified as asymmetrical and symmetrical stretching vibrations respectively. The FT-Raman band 1455, $1369 \mathrm{~cm}^{-1}$ are observed scissoring and wagging modes of the $\mathrm{CH}_{2}$ group. These modes are computed at 1459 and $1364 \mathrm{~cm}^{-1}$ by DFT method. The $\mathrm{O}-\mathrm{H}$ stretching vibration is theoretically predicted at $3676 \mathrm{~cm}^{-1}$ (mode no. 1) by B3LYP method. The computed wavenumbers at $1343 \mathrm{~cm}^{-1}$ and $295 \mathrm{~cm}^{-1}$ gives the COH inplane and $\mathrm{CCOH}$ out-off plane bending vibrations respectively.

\subsubsection{Cyclopropyl ring vibrations}

The $\mathrm{CH}_{2}$ symmetrical and asymmetrical stretching vibrations observed at $3032-3005 \mathrm{~cm}^{-1}$ and $3119-3100 \mathrm{~cm}^{-1}$ by DFT method. The FT-IR band at $1407 \mathrm{~cm}^{-1}$ and computed wavenumbers at $1419,1415 \mathrm{~cm}^{-1}$ by DFT method are identified as scissoring $\mathrm{CH}_{2}$ vibrations of the Cyclopropyl ring. The C-C stretching vibrations of this ring has been identified at $1192 \mathrm{~cm}^{-1}$ in FT-IR and 1199,815 , $805 \mathrm{~cm}^{-1}$ by DFT calculation. The C-C-H inplane bending vibrations identified at 1152,1150 , $742 \mathrm{~cm}^{-1}$ by DFT method.

\subsection{NBO analysis}

In the NBO analysis, the electron wave functions are interpreted in terms of a set of occupied Lewis type (bond or lone pair) and a set of unoccupied non-Lewis (anti-bond or Rydberg) localized NBO orbitals. The delocalization of electron density (ED) between these orbitals corresponds to a stabilizing donor acceptor interaction. A useful aspect of the NBO method is that it gives information about interactions in both filled and virtual orbital spaces, which could enhance the analysis of intra- and intermolecular interactions.

The second-order Fock matrix was carried out to evaluate the donor acceptor interactions in the NBO basis. The interactions result in a loss of occupancy from the localized NBO of the idealized Lewis structure into an empty nonLewis orbital. For each donor (i) and acceptor (j), the stabilization energy $E_{(2)}$ associated with the delocalization $\mathrm{i} / \mathrm{j}$ is estimated as

$$
E_{2}=\Delta E_{i j}=q_{i} \frac{F(i, j)^{2}}{\varepsilon_{j}-\varepsilon_{i}}
$$

where $\mathrm{q}_{\mathrm{i}}$ is the donor orbital occupancy, $\varepsilon_{\mathrm{i}}$ and $\varepsilon_{\mathrm{j}}$ are diagonal elements and $\mathrm{F}(\mathrm{i}, \mathrm{j})$ is the offdiagonal NBO Fock matrix element.

The molecular interaction is formed by the orbital overlap between $\pi(\mathrm{C} 11-\mathrm{C} 16) \rightarrow \pi^{*}(\mathrm{~N} 8$ $\mathrm{C} 9), \pi^{*}(\mathrm{~N} 12-\mathrm{C} 13), \pi^{*}(\mathrm{~N} 14-\mathrm{C} 15)$ resulting intra molecular charge transfer with stabilization energy is about $18.07,8.69,32.98 \mathrm{kCal} / \mathrm{mol}$. The intramolecular hyper conjugate energy between $\sigma$ and $\sigma^{*}$ electrons was found as $5.99,5.72 \mathrm{kCal} / \mathrm{mol}$ due to interactions $\sigma(\mathrm{C} 18-\mathrm{C} 20) \rightarrow \sigma^{*}(\mathrm{C} 18-\mathrm{C} 19)$, $\sigma^{*}(\mathrm{C} 19-\mathrm{C} 20)$. As can be seen from this Table 3, NBO analysis revealed that the $\pi^{*}(\mathrm{~N} 8-\mathrm{C} 9)$ $\rightarrow \pi^{*}(\mathrm{C} 11-\mathrm{C} 16), \pi^{*}(\mathrm{C} 11-\mathrm{C} 16), \pi^{*}(\mathrm{~N} 12-\mathrm{C} 13) \rightarrow \pi^{*}(\mathrm{C} 11-\mathrm{C} 16)$ and $\pi^{*}(\mathrm{~N} 14-\mathrm{C} 15) \rightarrow \pi^{*}(\mathrm{C} 11-\mathrm{C} 16)$ interactions give a strong stabilization to the system of the title compound by $69.21,124.25$ and $204.67 \mathrm{kCal} / \mathrm{mol}$ respectively. The interaction between lone pair $\mathrm{n}(\mathrm{N} 21)$ and the antibonding orbital $\mathrm{LP}(1) \mathrm{N} 21 \rightarrow \pi^{*}(\mathrm{~N} 12-\mathrm{C} 13)$ with stabilization energy $42.25 \mathrm{kCal} / \mathrm{mol}$. 
Table 3. Second order Perturbation theory analysis of Fock Matrix in NBO basis for Abacavir.

\begin{tabular}{|c|c|c|c|c|c|c|}
\hline Donor (i) & $E_{D}(i)(e)$ & Acceptor(j) & $E_{D}(j)(e)$ & $\begin{array}{c}\mathrm{E}^{(2) \mathrm{a}} \mathrm{KJ} \\
\mathrm{mol}^{-1}\end{array}$ & $\begin{array}{c}E(j)-E(i)^{b} \\
\text { a.u }\end{array}$ & $F(i, j)^{c}$ a.u \\
\hline$\sigma(\mathrm{C} 6-\mathrm{H} 28)$ & 1.965 & $\sigma^{*}(\mathrm{C} 9-\mathrm{N} 10)$ & 0.042 & 5.05 & 0.95 & 0.062 \\
\hline$\sigma(\mathrm{N} 8-\mathrm{C} 9)$ & 1.984 & $\sigma^{*}(\mathrm{C} 15-\mathrm{C} 16)$ & 0.041 & 6.76 & 1.38 & 0.087 \\
\hline$\pi(\mathrm{N} 8-\mathrm{C} 9)$ & 1.905 & $\pi^{*}(\mathrm{C} 11-\mathrm{C} 16)$ & 0.463 & 15.72 & 0.34 & 0.072 \\
\hline$\sigma(\mathrm{N} 8-\mathrm{C} 16)$ & 1.975 & $\sigma^{*}(\mathrm{C} 9-\mathrm{H} 31)$ & 0.020 & 5.55 & 1.23 & 0.074 \\
\hline$\sigma(\mathrm{C} 9-\mathrm{N} 10)$ & 1.984 & $\sigma^{*}(\mathrm{C} 11-\mathrm{N} 12)$ & 0.018 & 5.65 & 1.33 & 0.077 \\
\hline$\sigma(\mathrm{C} 11-\mathrm{C} 16)$ & 1.969 & $\sigma^{*}(\mathrm{C} 6-\mathrm{N} 10)$ & 0.044 & 5.63 & 1.03 & 0.068 \\
\hline \multirow[t]{3}{*}{$\pi(\mathrm{C} 11-\mathrm{C} 16)$} & 1.648 & $\pi^{*}(\mathrm{~N} 8-\mathrm{C} 9)$ & 0.335 & 18.07 & 0.26 & 0.061 \\
\hline & & $\pi^{*}(\mathrm{~N} 12-\mathrm{C} 13)$ & 0.463 & 8.69 & 0.26 & 0.044 \\
\hline & & $\pi^{*}(\mathrm{~N} 14-\mathrm{C} 15)$ & 0.466 & 32.98 & 0.26 & 0.086 \\
\hline$\sigma(\mathrm{N} 12-\mathrm{C} 13)$ & 1.979 & $\sigma^{*}(\mathrm{~N} 10-\mathrm{C} 11)$ & 0.044 & 8.27 & 1.28 & 0.092 \\
\hline \multirow[t]{2}{*}{$\pi(\mathrm{N} 12-\mathrm{C} 13)$} & 1.772 & $\pi^{*}(\mathrm{C} 11-\mathrm{C} 16)$ & 0.463 & 31.52 & 0.32 & 0.096 \\
\hline & & $\pi^{*}(\mathrm{~N} 14-\mathrm{C} 15)$ & 0.466 & 5.53 & 0.30 & 0.039 \\
\hline$\pi(\mathrm{N} 14-\mathrm{C} 15)$ & 1.763 & $\pi^{*}(\mathrm{~N} 12-\mathrm{C} 13)$ & 0.463 & 36.88 & 0.30 & 0.100 \\
\hline$\sigma(\mathrm{N} 17-\mathrm{H} 32)$ & 1.981 & $\sigma^{*}(\mathrm{~N} 14-\mathrm{C} 15)$ & 0.016 & 5.23 & 1.19 & 0.070 \\
\hline \multirow{2}{*}{$\sigma(\mathrm{C} 18-\mathrm{C} 19)$} & 1.956 & $\sigma^{*}(\mathrm{C} 18-\mathrm{C} 20)$ & 0.028 & 5.75 & 0.88 & 0.064 \\
\hline & & $\sigma^{*}(\mathrm{C} 19-\mathrm{C} 20)$ & 0.021 & 5.33 & 0.88 & 0.061 \\
\hline \multirow[t]{2}{*}{$\sigma(\mathrm{C} 18-\mathrm{C} 20)$} & 1.957 & $\sigma^{*}(\mathrm{C} 18-\mathrm{C} 19)$ & 0.039 & 5.99 & 0.89 & 0.065 \\
\hline & & $\sigma^{*}(\mathrm{C} 19-\mathrm{C} 20)$ & 0.021 & 5.72 & 0.88 & 0.064 \\
\hline \multirow[t]{2}{*}{$\sigma(\mathrm{C} 19-\mathrm{C} 20)$} & 1.963 & $\sigma^{*}(\mathrm{C} 18-\mathrm{C} 19)$ & 0.039 & 5.17 & 0.88 & 0.060 \\
\hline & & $\sigma^{*}(\mathrm{C} 18-\mathrm{C} 20)$ & 0.028 & 5.67 & 0.87 & 0.063 \\
\hline $\mathrm{LP}(2) \mathrm{O} 2$ & 1.954 & $\sigma^{*}(\mathrm{C} 1-\mathrm{C} 3)$ & 0.037 & 8.04 & 0.67 & 0.066 \\
\hline \multirow[t]{2}{*}{ LP(1)N8 } & 1.926 & $\sigma^{*}(\mathrm{C} 9-\mathrm{N} 10)$ & 0.042 & 9.72 & 0.80 & 0.079 \\
\hline & & $\sigma^{*}(\mathrm{C} 11-\mathrm{C} 16)$ & 0.044 & 6.84 & 0.93 & 0.072 \\
\hline \multirow[t]{3}{*}{ LP(1)N10 } & 1.582 & $\sigma^{*}(\mathrm{C} 6-\mathrm{C} 7)$ & 0.023 & 6.73 & 0.60 & 0.063 \\
\hline & & $\pi^{*}(\mathrm{~N} 8-\mathrm{C} 9)$ & 0.335 & 45.23 & 0.27 & 0.100 \\
\hline & & $\pi^{*}(\mathrm{C} 11-\mathrm{C} 6)$ & 0.463 & 40.85 & 0.29 & 0.098 \\
\hline \multirow[t]{2}{*}{ LP(1)N12 } & 1.901 & $\sigma^{*}(\mathrm{C} 11-\mathrm{C} 16)$ & 0.044 & 10.67 & 0.90 & 0.089 \\
\hline & & $\sigma^{*}(\mathrm{C} 13-\mathrm{N} 14)$ & 0.036 & 13.14 & 0.86 & 0.096 \\
\hline \multirow[t]{2}{*}{ LP(1)N14 } & 1.900 & $\sigma^{*}(\mathrm{~N} 12-\mathrm{C} 13)$ & 0.036 & 13.79 & 0.85 & 0.098 \\
\hline & & $\sigma^{*}(\mathrm{C} 15-\mathrm{C} 16)$ & 0.041 & 10.39 & 0.88 & 0.087 \\
\hline \multirow[t]{3}{*}{ LP(1)N17 } & 1.724 & $\pi^{*}(\mathrm{~N} 14-\mathrm{C} 15)$ & 0.466 & 57.31 & 0.26 & 0.116 \\
\hline & & $\sigma^{*}(\mathrm{C} 18-\mathrm{C} 19)$ & 0.039 & 5.59 & 0.60 & 0.055 \\
\hline & & $\sigma^{*}(\mathrm{C} 18-\mathrm{H} 33)$ & 0.030 & 7.62 & 0.73 & 0.071 \\
\hline LP(1)N21 & 1.800 & $\pi^{*}(\mathrm{~N} 12-\mathrm{C} 13)$ & 0.463 & 42.25 & 0.28 & 0.104 \\
\hline$\pi^{*}(\mathrm{~N} 8-\mathrm{C} 9)$ & 0.335 & $\pi^{*}(\mathrm{C} 11-\mathrm{C} 16)$ & 0.463 & 69.21 & 0.02 & 0.057 \\
\hline$\pi^{*}(\mathrm{~N} 12-\mathrm{C} 13)$ & 0.463 & $\pi^{*}(\mathrm{C} 11-\mathrm{C} 16)$ & 0.463 & 124.25 & 0.02 & 0.071 \\
\hline$\pi^{*}(\mathrm{~N} 14-\mathrm{C} 15)$ & 0.466 & $\pi^{*}(\mathrm{C} 11-\mathrm{C} 16)$ & 0.463 & 204.67 & 0.02 & 0.082 \\
\hline
\end{tabular}

$\mathrm{E}_{\mathrm{D}}$-means Electron Density

${ }^{a} E^{(2)}$ - means energy of hyper conjugative interactions

${ }^{b}$ Energy difference between donor and acceptor $i$ and $j$ NBO orbitals

${ }^{c} \mathrm{~F}(\mathrm{i}, \mathrm{j})$ is the Fock matrix element between $\mathrm{i}$ and $\mathrm{j}$ NBO orbitals

\subsection{UV-Visible spectral studies}

The highest occupied molecular orbitals (HOMOs) and the lowest-lying unoccupied molecular orbitals (LUMOs) are named as frontier molecular orbitals (FMOs). The FMOs play an important role in the optical and electric properties, as well as in quantum chemistry and UV-VIS spectra [26]. Absorption maxima $(\lambda \max )(\mathrm{nm})$ for lower-lying singlet states of the molecule of the molecule have been calculated by TD-DFT/B3LYP method. The computed properties such as 
absorption wavelength $(\lambda)$, excitation energies $(E)$, frontier molecular orbital energies, and oscillator strengths (f) are listed in Table 4. Fig. 4 shows the observed UV-Vis spectra of title compound in ethanol solvent. For TD-DFT calculations, the theoretical absorption band was predicted at 240.18 $\mathrm{nm}$ with oscillator strength being 0.0906 in ethanol solvent and at $239.68 \mathrm{~nm}$ with oscillating strength 0.0765 in gas phase can easily be seen that this corresponds to the experimental absorption at $240 \mathrm{~nm}$. Fig. $5(\mathrm{a}, \mathrm{b})$ shows the distributions and energy levels of the HOMOs and LUMOs orbitals computed at the B3LYP/6-31G(d,p) level for Abacavir. The conjugated molecules are described by a small HOMO-LUMO separation which is the consequence of a significant amount of intra molecular charge transfer from electron donor groups to the capable electron acceptor groups through $\pi$-conjugated path [27]. The calculated energy values of HOMO and LUMO are $-5.1506 \mathrm{eV}$ and $-0.1474 \mathrm{eV}$ and the frontier orbital energy gap value is $-5.0032 \mathrm{eV}$ for Abacavir.

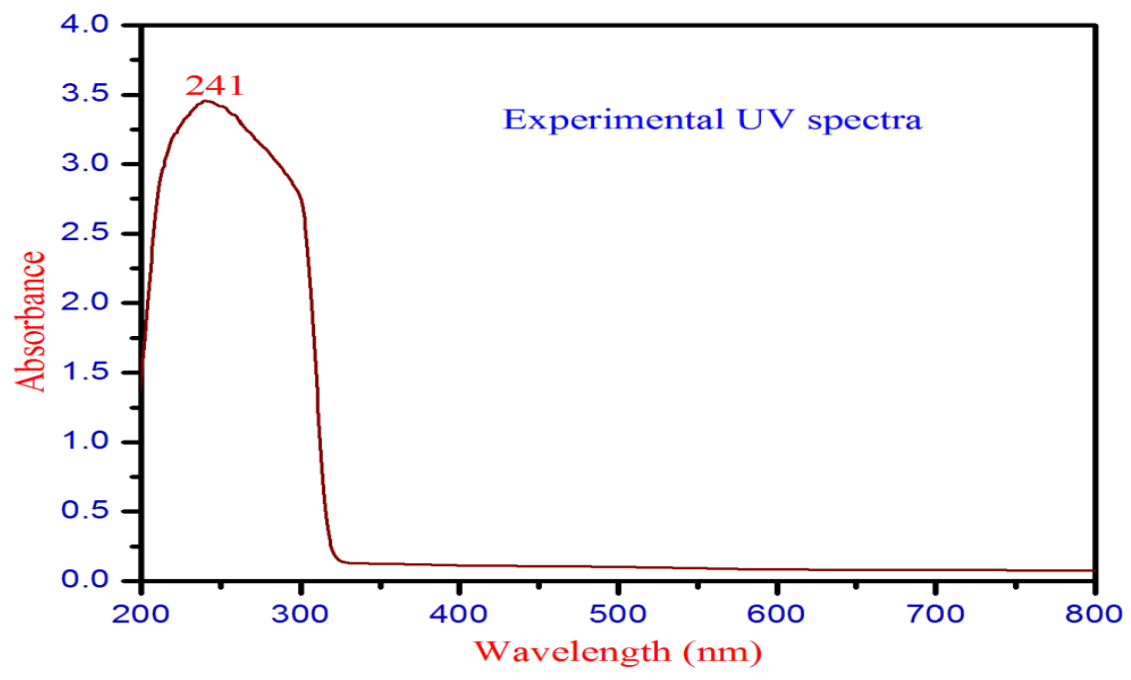

Figure 4. UV-Visible spectrum (Ethanol) of Abacavir.

Table 4. The experimental and computed absorption wavelength $\lambda(\mathrm{nm})$, excitation energies $\mathrm{E}(\mathrm{eV})$, absorbance and oscillator strengths $(f)$ of Abacavir in Ethanol solution and gas phase.

\begin{tabular}{|c|c|c|c|c|c|c|c|}
\hline \multirow{2}{*}{\multicolumn{2}{|c|}{$\begin{array}{c}\text { Experimental } \\
\text { Ethanol }\end{array}$}} & \multicolumn{6}{|c|}{ TD-DFT/B3LYP/6-31G(d,p) } \\
\hline & & \multicolumn{3}{|c|}{ Ethanol } & \multicolumn{3}{|c|}{ Gas } \\
\hline$\lambda(\mathrm{nm})$ & Abs. & $\lambda(\mathrm{nm})$ & $\mathrm{E}(\mathrm{eV})$ & f(a.u) & $\lambda(\mathrm{nm})$ & $\mathrm{E}(\mathrm{eV})$ & f(a.u) \\
\hline & & 266.66 & 4.6496 & 0.3502 & 274.90 & 4.5101 & 0.0599 \\
\hline & & 244.34 & 5.0743 & 0.0305 & 259.43 & 4.7791 & 0.2455 \\
\hline 240 & 3.4571 & 240.18 & 5.1622 & 0.0906 & 239.68 & 5.1730 & 0.0765 \\
\hline
\end{tabular}




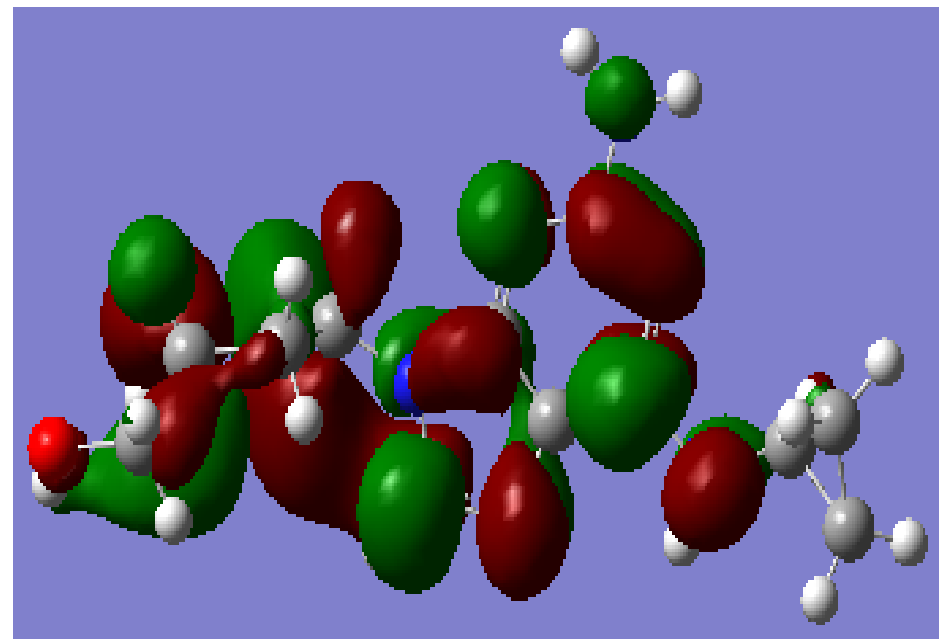

(a) $\mathrm{LUMO}=-0.1474 \mathrm{eV}$

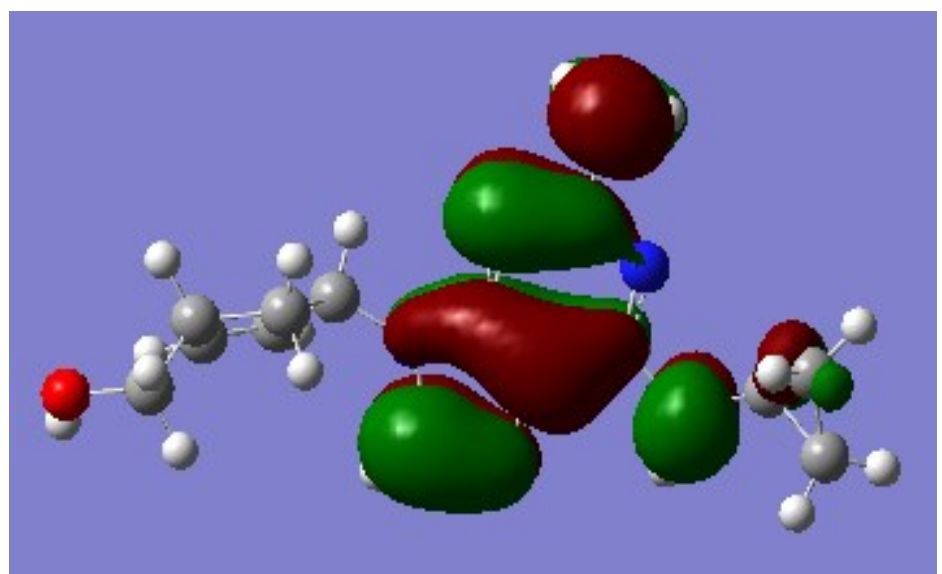

(b) $\mathrm{HOMO}=-5.1506 \mathrm{eV}$

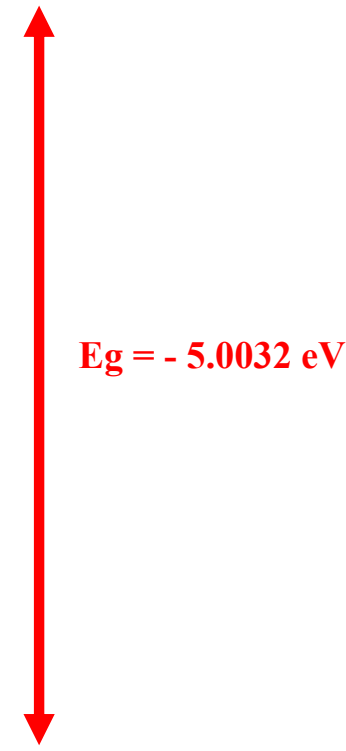

Figure $5(\mathbf{a}, \mathbf{b})$. The atomic orbital compositions of the frontier molecular orbital for Abacavir.

\subsection{Molecular electro static potential analysis}

MEP is pertained to the electronic density and is a very useful descriptor in understanding sites for nucleophilic reaction and electrophilic attack as well as hydrogen bonding interactions [28]. It also provides visual understanding of relative polarity of the molecule. The MEP surface has been plotted for the molecules Abacavir in Fig. 6. From the Fig.6 it can be seen that region of negative charge is pictured out in red colour and it is found around the electronegative $\mathrm{N}$ in the purine ring and $\mathrm{O} 20$ atom in the hydroxy groups in the molecule Abacavir. The red region gives the electrophilic attack. The blue colour region represents strong positive region (all hydrogen atoms) and is prone to nucleophilic attack. 


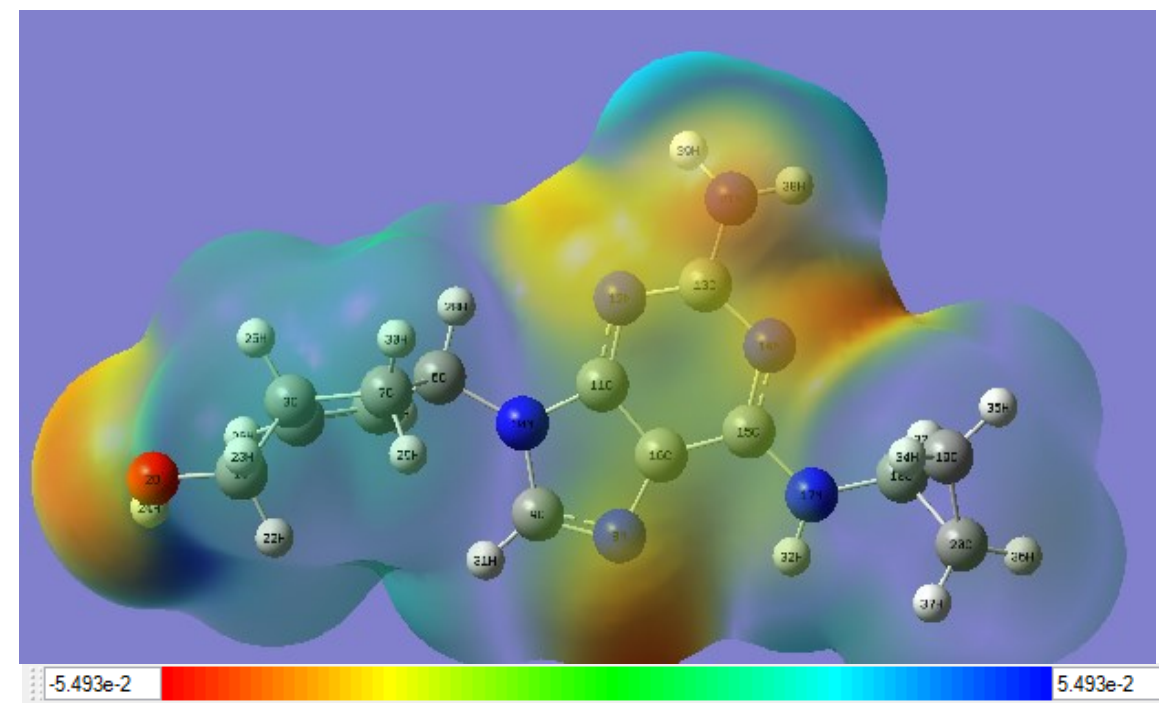

Figure 6. Molecular electro static potential Map of Abacavir.

\subsection{Molecular docking studies}

Molecular docking studies were performed to investigate the binding affinities of the title compound $\mathrm{ABC}$ and the human protein Abacavir, PDB ID: 3VRI [29]. The ligand-protein complex stability was successfully made by some features such as hydrogen bond interactions, vander Waals forces, $\pi-\pi$ stacking, hydrophilic and hydrophobic interactions. High resolution crystal structure of Abacavir compound hydrogen and water molecules are reductase was downloaded from the protein data bank website (PDB ID: 3VRI) and all molecular docking calculations were performed on Auto Dock-Vina software [30]. The protein was prepared for docking by removing the co-crystallized ligands, waters and co-factors and the Auto Dock Tools graphical user interface was used to calculate Kollman charges and polar hydrogens. Molecular docking studies were performed to investigate the higher binding affinities and total intermol energy of the newly compound Abacavir is $-7.74 \mathrm{kcal} / \mathrm{mol}$ and $-5.19 \mathrm{kcal} / \mathrm{mol}$ lower binding affinities and total intermol energy of the title molecules is $-9.16 \mathrm{kcal} / \mathrm{mol}$ and $-6.62 \mathrm{kcal} / \mathrm{mol}$ respectively. The hydrophobic interactions between ABC and TYR 74, ASP114, Gly-217, SCR116 is found at $2.81 \AA, 3.04 \AA$, and $3.12 \AA$. On the other hand, a $\pi-\pi$ stacking exists between phenyls of ABC and TRP147 is $3.47 \AA$. The title molecule is given in Fig. 7 and the values are tabulated in Table 5.

Table 5. Comparison of Est. Free Energy of Binding, Total Intermol. Energy of Abacavir.

\begin{tabular}{|c|l|l|}
\hline Mode & Est. Free Energy of Binding & Total Intermol. Energy \\
\hline 1 & $-7.74 \mathrm{kcal} / \mathrm{mol}$ & $-9.16 \mathrm{kcal} / \mathrm{mol}$ \\
\hline 2 & $-7.39 \mathrm{kcal} / \mathrm{mol}$ & $-8.86 \mathrm{kcal} / \mathrm{mol}$ \\
\hline 3 & $-7.21 \mathrm{kcal} / \mathrm{mol}$ & $-8.61 \mathrm{kcal} / \mathrm{mol}$ \\
\hline 4 & $-5.43 \mathrm{kcal} / \mathrm{mol}$ & $-6.79 \mathrm{kcal} / \mathrm{mol}$ \\
\hline 5 & $-5.19 \mathrm{kcal} / \mathrm{mol}$ & $-6.62 \mathrm{kcal} / \mathrm{mol}$ \\
\hline
\end{tabular}



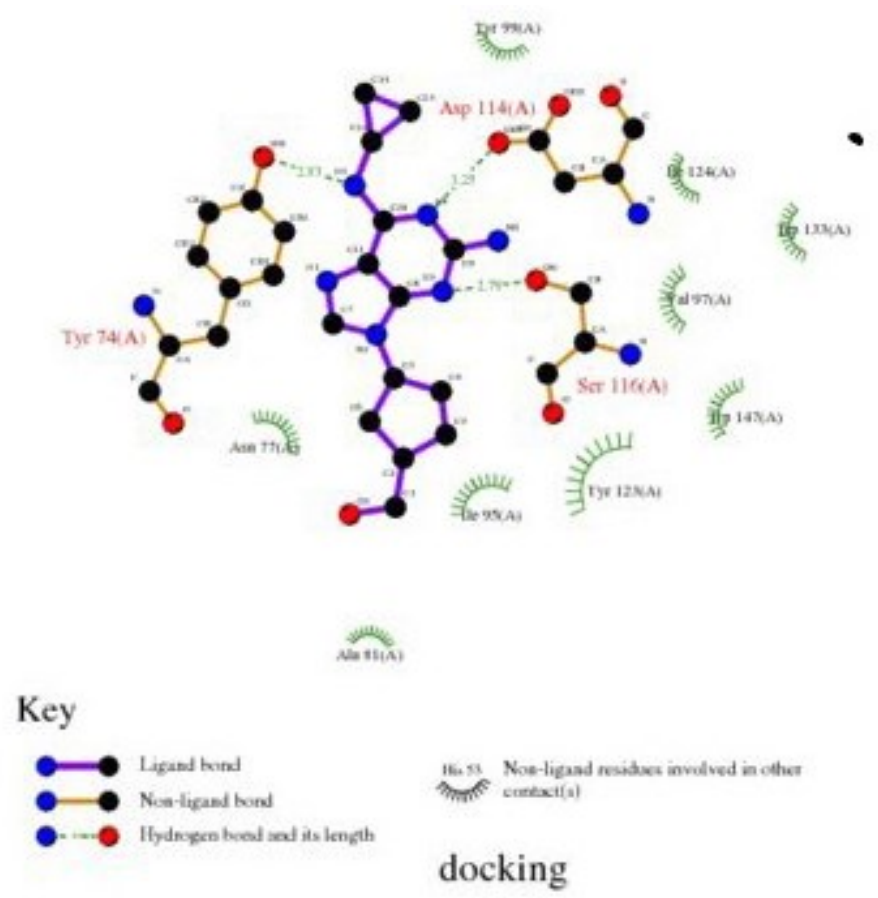

Figure 7a. Hydrogen bonds between docked glutathione and amino acids residues of Abavacir.

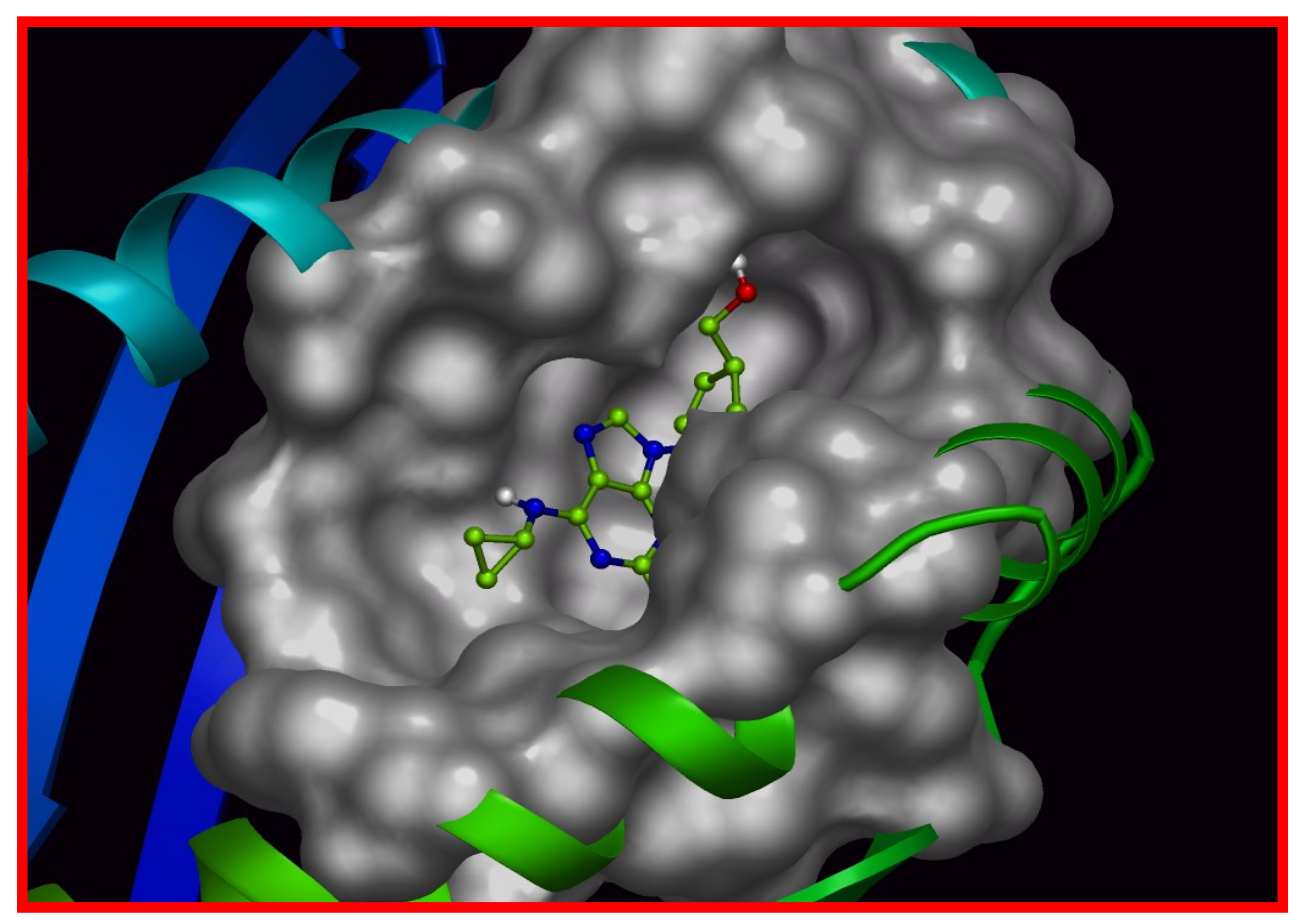

(b) 


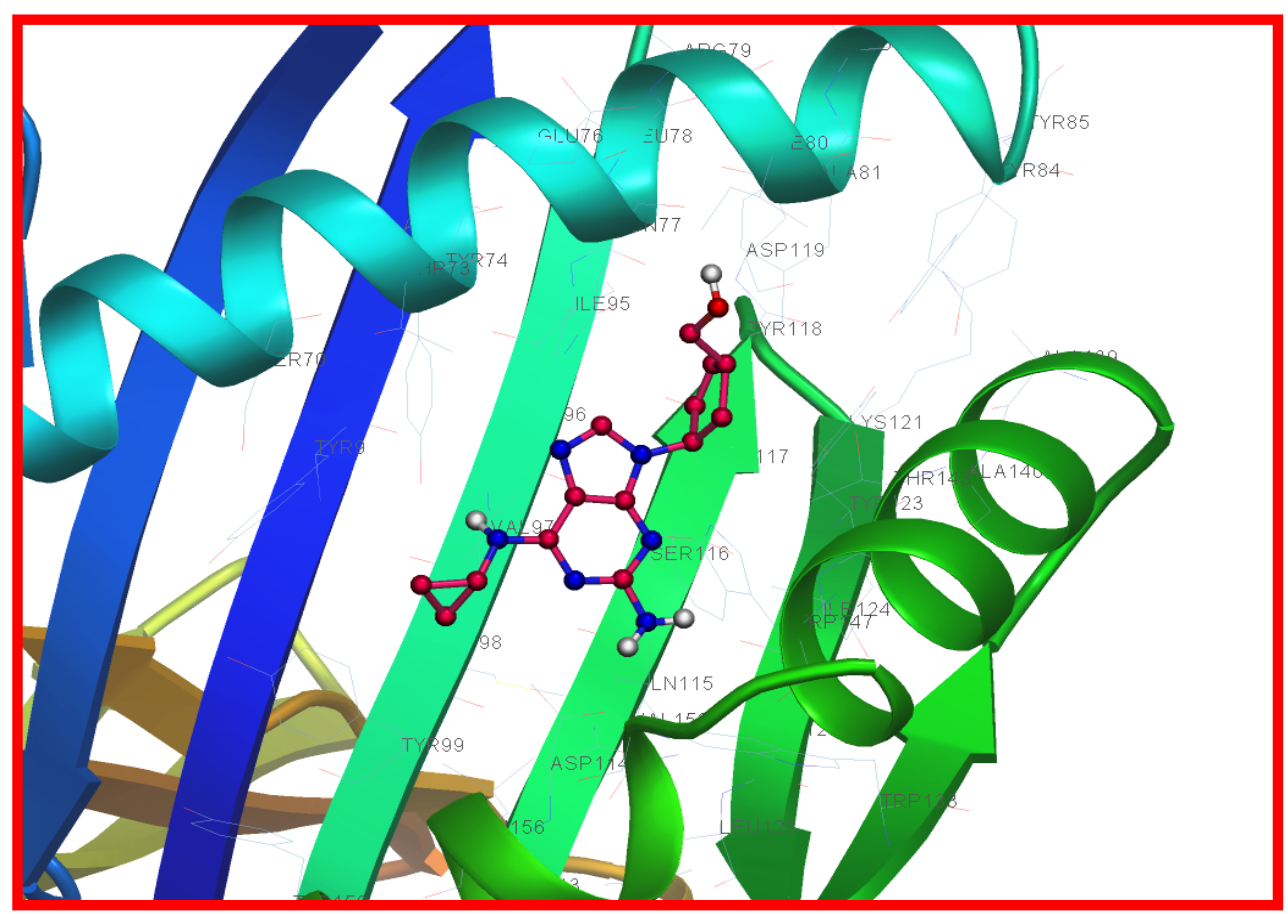

(c)

Figure $7(\mathbf{b}, \mathbf{c})$. Docked conformation of ligand in the binding site of 3 VRI.

\section{Conclusions}

This paper presents spectroscopic and molecular modeling studies on the interaction of Abacavir using, FT-IR, FT-Raman, UV-Vis spectrum. The equilibrium geometries, HOMOLUMO analysis and vibrational frequencies of Abacavir have been calculated at B3LYP levels using 6-31G(d,p) basis set. The title molecular HOMO-LUMO energy gap is $-5.0032 \mathrm{eV}$. The agreement between the optimized and experimental crystal structure is quite good, which shows that the geometry optimization almost exactly reproduces the experimental conformation. The electronic properties are also calculated and compared with the experimental UV-Vis spectrum. The fundamental vibrational modes of the title compound have been precisely assigned and analyzed and the experimental results were compared with the theoretical values. A good agreement between experimental and calculated normal modes of vibrations has been observed. The NBO analysis indicates the intramolecular charge transfer between the bonding and antibonding orbitals. Molecular docking study has been performed by in silico method to analysis their HIVaspects against Abacavir carrier protein. Hydrogen bonds between docked Abacavir and amino acids residues of protein. Docked conformation of ligand in the binding site of Abacavir with 3VRI respectively. 


\section{References}

[1] P. Kommavarapu et al., Simultaneous estimation of degree of crystallinity in combination drug product of abacavir, lamivudine and neverapine using X-ray powder diffraction technique, Journal of Young Pharmacists. 5(4) (2013) 127-132.

[2] S.K. Gangwer, A.K. Srivastava, R.B. Singh, Experimental [FT-IR, UV-Visible, NMR] spectroscopy and molecular structure, global reactivity parameters, Fukui Function, NBO, NLO, Homo-Lmo, MESP, and QTAM analysei of abacavir using density functional theory, IOSR-journal of Biotechnology and biochemistry. 2 (2016) 19-34.

[3] P. Echeverría, E. Negredo, G. Carosi, Similar antiviral efficacy and tolerability between efavirenz and lopinavir/ritonavir, administered with abacavir/lamivudine (Kivexa), in antiretroviral-naïve patients: A 48-week, multicentre, randomized study (Lake Study), Antiviral Research. 85 (2010) 403-408.

[4] Gaussian Inc., Gaussian 03 Program, Gaussian Inc., Wallingford, 2004.

[5] H.B. Schlegel, Optimization of equilibrium geometries and transition structures, J. Comput. Chem. 3 (1982) 214-218.

[6] A. Frisch, A.B. Nielson, A.J. Holder, Gaussview user manual, Gaussian Inc., Pittsburgh, PA, 2000.

[7] M.H. Jamróz, Vibrational Energy Distribution Analysis, VEDA 4, Warsaw, 2004.

[8] N.C. Handy, P.E. Maslen, R.D. Amos, Study of methane, acetylene, ethene, and benzene using Kohn-Sham theory, J. Phys. Chem. 97 (1993) 4392-4396.

[9] V. Krishnakumar, R. Mathammal, S. Muthunatesan, FT-IR and Raman spectra vibrational assignments and density functional calculations of 1-naphthyl acetic acid, Spectrochim. Acta A. 70 (2008) 210-216.

[10] E.D. Glendening et al., NBO Version3.1, TCI, University of Wisconsin, Madison, 1998.

[11] L. E. Sutton, Tables of interatomic distances, Chemical Society, London, 1958.

[12] K. Govindarasu, E. Kavitha, Vibrational spectra, molecular structure, NBO, NMR, UV, first order hyperpolarizability, analysis of (S)-(-)-N-(5-Nitro-2-pyridyl) alaninol by Density functional theory, Spectrochim. Acta A. 127 (2014) 498-510.

[13] P.T. Illing et al., Immune self-reactivity triggered by drug-modified HLA-peptide repertoire, Nature. 486 (2012) 554-558.

[14] M.F. Sanner, Python: a programming language for software integration and development, Journal of Molecular Graphics and Modelling. 17 (1999) 57-61.

[15] A.A. Adeniyi, P.A. Ajibade, Inhibitory activities and possible anticancer targets of Ru(II)based complexes using computational docking method, Journal of Molecular Graphics and Modelling. 38 (2012) 60-69.

[16] R. Thomsen, M.H. Christensen, MolDock: A New Technique for High-Accuracy Molecular Docking, J. Med. Chem. 55 (2012) 623-638.

[17] P.TT. Pham, Abacavir methanol 2.5-solvate, Acta Crystallographica Section E: Structure Reports Online. 8 (2009) o193.

[18] A.A. Al-Saadi, J. Laane, Ab initio and DFT calculations for the structure and vibrational spectra of cyclopentene and its isotopomers, J. Mol. Struct. 830 (2007) 46-57.

[19] Ismat Fatima et al., 6-Benzylsulfanyl-9H-purine, Acta Cryst. E. 65 (2009) o2994. 
[20] C.S.C. Kumara et al., Synthesis, molecular structure, spectroscopic characterization and quantum chemical calculation studies of (2E)-1-(5-chlorothiophen-2-yl)-3-(2,3,4trimethoxyphenyl)prop-2-en-1-one, J. Mol. Struct. 1085 (2015) 63-77.

[21] V. Balachandran, K. Parimala, Automeric purine forms of 2-amino-6-chloropurine (N9H10 and N7H10): Structures, vibrational assignments, NBO analysis, hyperpolarizability, HOMOLUMO study using B3 based density functional calculations, Spectrochimica Acta Part A. 96 (2012) 340-351.

[22] K. Parimala, V. Balachandran, Vibrational spectroscopic (FTIR and FT Raman) studies, first order hyperpolarizabilities and HOMO, LUMO analysis of p-toluenesulfonyl isocyanate using ab initioHF and DFT methods, Spectrochim. Acta A. 81 (2011) 711-723.

[23] M. Silverstein, G.C. Basseler, T.C. Morrill, Spectrometric identification of organic compound, Wiley, New York, 1981.

[24] G. Socrates, Infrared and Raman characteristic group frequencies, Tables and Charts, Third ed., vol. 2, John Wiley, Chichester, 2001.

[25] N.B. Colthup, L.H. Daly, S.E. Wiberley, Introduction to infrared and Raman spectroscopy, Academic Press, New York, 1990.

[26] I. Fleming, Frontier orbitals and organic chemical reactions, Wiley, London, 1976.

[27] C.H. Choi, M. Kertesz, Conformational information from vibrational spectra of styrene, transstilbene, and cis-stilbene, J. Phys. Chem. A. 101 (1997) 3823-3831.

[28] N. Okulik, A.H. Jubert, Theoretical analysis of the reactive sites of non-steroidal antiinflammatory drugs, Internet Electron J. Mol. Des. 4 (2005) 17-30.

[29] N. Strushkevich, S.A. Usanov, H.W. Park, Structural basis of human CYP51 inhibition by antifungal azoles, J. Mol. Biol. 397 (2010) 1067-1078.

[30] O. Trott, A.J. Olson, AutoDock Vina: improving the speed and accuracy of docking with a new scoring function, efficient optimization and multithreading, J. Comput. Chem. 31 (2010) 455-461. 